\title{
Tensor-based methods for numerical homogenization from high-resolution images ${ }^{\text {ts }}$
}

\author{
L. Giraldi, A. Nouy*, G. Legrain, P. Cartraud \\ LUNAM Université, GeM, UMR CNRS 6183, École Centrale de Nantes, Université de Nantes, France
}

\begin{abstract}
We present a complete numerical strategy based on tensor approximation techniques for the solution of numerical homogenization problems with geometrical data coming from high resolution images. We first introduce specific numerical treatments for the translation of image-based homogenization problems into a tensor framework. It includes the tensor approximations in suitable tensor formats of fields of material properties or indicator functions of multiple material phases recovered from segmented images. We then introduce some variants of Proper Generalized Decomposition (PGD) methods for the construction of tensor decompositions in different tensor formats of the solution of boundary value problems. A new definition of PGD is introduced which allows the progressive construction of a Tucker decomposition of the solution. This tensor format is well adapted to the present application and improves convergence properties of tensor decompositions. Finally, we use a dual-based error estimator on quantities of interest which was recently introduced in the context of PGD. We exhibit its specificities when it is used for assessing the error on the homogenized properties of the heterogeneous material. We also provide a complete goal-oriented adaptive strategy for the progressive construction of tensor decompositions (of primal and dual solutions) yielding to predictions of homogenized quantities with a prescribed accuracy.
\end{abstract}

Keywords: Image-based computing, Numerical Homogenization, Tensor methods, Proper Generalized Decomposition (PGD), Model Reduction, Goal-oriented error estimation, Adaptive approximation

\section{Introduction}

With the development of affordable high resolution imaging techniques, such as X-ray microtomography, high resolution geometrical characterization of material microstructures is increasingly used in industry. However, the amount of informations that are available is still difficult to handle in numerical models. This is why dedicated approaches

\footnotetext{
This work is supported by the French National Research Agency (grant ANR-2010-COSI-006-01).

* Corresponding Author

Email addresses: Loic.Giraldi@ec-nantes.fr (L. Giraldi), Anthony.Nouy@ec-nantes.fr (A.

Nouy), Gregory.Legrain@ec-nantes.fr (G. Legrain), Patrice.Cartraud@ec-nantes.fr (P. Cartraud)

Preprint submitted to Computer Methods in Applied Mechanics and Engineering October 27, 2012
} 
have been proposed in order to incorporate these informations for simulation purposes [57]. The most used approach in this context is the voxel-based finite element method introduced in $[21,25]$, where each voxel of the model is transformed into a finite element. The approach is straightforward and automatic for the generation of the computational model (see [43] for a review). However, it leads to huge numerical models, as the number of elements corresponds to the number of voxels in the image (in the order of 8 billion of elements for a full resolution $2000 \times 2000 \times 2000$ voxels CT scan). In addition, the representation of the interfaces is not smooth, which induces local oscillations in the mechanical fields $[9,40,53]$. The size of the model can be decreased with the use of an octree coarsening away from the interfaces [40] or by decreasing the resolution of the image [3, 38, 42]. However, this can severely decrease the geometrical accuracy (more jagged interfaces) and increase the oscillations. In order to get rid of these oscillations, mesh smoothing techniques can be considered, e.g. [6]. Ultimately, full resolution images can still be considered, using Fast Fourier Transforms (FFT) algorithms [44] in the case of periodic problems.

A second class of approaches consists in extracting the material interfaces from the image and then in constructing an unstructured conforming mesh from these informations, e.g. [41, 56, 57]. This allows to generate smooth interfaces and adapt the mesh in order to master the size of the model. However, meshing complex geometries is still difficult and usually requires human guidance.

Finally, non-conforming approaches can be considered (see [13, 54] among others): these approaches allows to avoid meshing issues. In particular, the eXtended Finite Element Method (X-FEM) has been used by the authors for the treatment of $2 \mathrm{D}$ and $3 \mathrm{D}$ image-based analysis $[35,36,40]$. An integrated approach was proposed in order to incorporate the geometrical informations into the numerical model. It is based on the use of Level-set functions [51], for both segmentation and mechanical analysis. Thanks to the use of tailored enrichment functions, it is possible to represent the interfaces on a nonconforming mesh. The size of the numerical model is decreased thanks to the use of an octree database that enables to keep maximum geometrical accuracy near the interfaces. This allows to obtain a good compromise between easy mesh generation and accuracy (both geometrical and mechanical). More recently, an improvement was proposed by the use of a high-order two mesh strategy that enables high geometrical and mechanical accuracy on coarse meshes [37].

Despite of the improvements in the numerical efficiency of the methods discussed above, image-based computations are still computationaly demanding, leading to time consuming studies especially for large resolution images. There is still a need for new approaches that would allow the efficient resolution of such large scale problems.

This is why an alternative path is proposed in this paper. It relies on the use of tensor approximation methods for the solution of image-based homogenization problems. The basic idea is to interpret 2 or 3 -dimensional fields as 2 or 3 -order tensors, and to use tensor approximation methods for the approximate solution of boundary value problems. The use of suitable tensor formats allows to drastically reduce the computational costs (time and memory storage) and therefore allows the computation on very high resolution images. This paper provides a complete tensor-based numerical methodology, going from the translation of homogenization problems into a tensor framework, to the development of a goal-oriented adaptive construction of tensor decompositions based on error 
estimation methods, and dedicated to the present application.

We first translate image-based homogenization problems to a tensor framework by introducing suitable tensor approximations of geometrical data. Tensor approximation methods are applied to indicator functions of material phases, which are previously smoothed in order to improve the convergence properties of their decompositions. Suitable weak formulations of boundary value problems preserving tensor format are introduced in order to handle the different types of boundary conditions that are used in classical numerical homogenization methods. Regarding the construction of tensor approximations of the solution of PDEs, we use Proper Generalized Decomposition methods (PGD), which is a family of methods for the construction of tensor decompositions without a priori information on the solution of the PDE [10, 28, 45, 46] (see [12] for a short review on PGD methods). Theoretical convergence properties have been recently obtained for a class of PGD algorithms [7, 15, 16]. Note that a basic PGD algorithm has been used in [11] for the numerical solution of PDEs with heterogeneous materials whose geometry is easily represented in a tensor format. The method has also been used for deriving efficient non-concurrent non-linear homogenization strategies [31].

Although PGD methods rely on general concepts in approximation of tensors, practical algorithms have only been provided for the approximation in canonical tensor format. However, this format is known to have bad topological properties yielding ill-posedness of best approximation problems in the set of rank- $r$ canonical tensors for $d>2$ and $r>1$ [52]. Greedy constructions of canonical tensor decompositions allow to circumvent this issue but present only poor convergence properties. Here, we introduce variants of PGD algorithms for the construction of tensor decompositions in different tensor formats. In particular, we introduce a new definition of PGD which allows the progressive construction of a Tucker approximation of the solution. This tensor format is well adapted to the present application and yields to improved convergence properties of tensor decompositions. The subset of Tucker tensors with bounded rank is known to possess nice topological properties yielding well posedness and numerical stability of best approximation problems in these subsets [14]. Moreover, efficient algorithms based on SVD have been proposed for computing quasi-optimal Tucker approximations of a tensor, with controlled precision [34]. The algorithm proposed in this paper can be interpreted as an adaptive subspace-based model reduction method which consists in constructing a sequence of reduced approximation spaces extracted from successive rank-one corrections. An approximation (in Tucker format) is then obtained by a projection on the tensor product of these approximation spaces. The main drawback of the use of Tucker tensors is that it suffers from the curse of dimensionality. For dimension $d>3$, a recent format coined "hierarchical Tucker tensors" [19] combines the advantages of the canonical and the Tucker tensors. This work is a first step toward the use of hierarchical Tucker tensors within the PGD.

We finally devise a goal-oriented error estimation strategy in order the assess the error on quantities of interest which are the homogenized properties. Error estimation methods have been first introduced in the context of PGD in $[2,29]$. Here, we use a classical dual-based error estimator (see [1]), which has been used in [2] in the context of PGD methods. The originality of the present contribution consists in providing a complete adaptive strategy for the progressive construction of tensor decompositions yielding to predictions of homogenized quantities with a prescribed accuracy. Note that the proposed adaptive strategy could also be used in other context for goal-oriented 
approximation of PDEs in tensor formats.

The outline is as follows: Section 1 presents the homogenization problems and their variational formulations. Section 2 introduces the tensor framework and notations used for separated representations. Then section 3 presents how the solution of PDEs can be approximated under separated representations with the PGD. In particular, we detail a new algorithm for the progressive construction of a Tucker decomposition. Next, image geometry and boundary conditions are expressed in a tensor format in section 4 . First numerical examples are introduced in section 5 . Then, in section 6 , we introduce a goaloriented adaptive algorithm using error estimators on homogenized properties. Finally, the article presents an application on a cast iron image extracted from a tomography, where we use the complete goal-oriented adaptive solution method.

\section{Homogenization problems and variational formulations}

In this section, we introduce classical homogenization methods for a linear heat diffusion problem. Homogenization problems are boundary value problems formulated on a domain $\Omega$ which constitutes a representative volume of an heterogeneous material. The solution of these problems allows to extract effective for apparent macroscopic properties of the material depending on whether $\Omega$ is larger than the representative volume element (RVE). Note however that the prediction of the size of the representative volume is out of the scope of this paper. The reader can refer to $[23,24,39,48]$ for methodologies to estimate the size of the representative volume. In the following, we will identify both apparent and homogenized properties.

\subsection{Scale transition and localization problems}

We denote by $u$ and $q$ the temperature and flux fields respectively. The macroscopic gradient of the field $\underline{\nabla} u^{M}$ and the macroscopic flux $q^{M}$ are defined through a spatial averaging of the corresponding microscopic quantities $\underline{\nabla} u$ and $\underline{q}$ over the representative volume $\Omega$ :

$$
\begin{aligned}
\underline{\nabla} u^{M} & =<\underline{\nabla} u>=\frac{1}{|\Omega|} \int_{\Omega} \underline{\nabla} u d \Omega \\
\underline{q}^{M} & =<\underline{q}>=\frac{1}{|\Omega|} \int_{\Omega} \underline{q} d \Omega
\end{aligned}
$$

The inverse process yielding the microscopic fields from the macroscopic ones is called localization. Given $\underline{\nabla} u^{M}$ or $\underline{q}^{M}$, microscopic fields $\underline{\nabla} u$ and $\underline{q}$ are obtained by solving localization problems which are boundary value problems defined on $\Omega$ :

$$
\left\{\begin{array}{l}
\underline{\nabla} \cdot \underline{q}(\underline{x})=0 \text { on } \Omega \\
\underline{q}(\underline{x})=-\underline{\underline{K}}(\underline{x}) \cdot \underline{\nabla} u(\underline{x}) \text { on } \Omega \\
+ \text { boundary conditions depending on } \underline{\nabla} u^{M} \text { or } \underline{q}^{M}
\end{array}\right.
$$

where $\underline{\underline{K}}$ is the conductivity field. If the data is the macroscopic gradient $\underline{\nabla} u^{M}$ or the macroscopic flux $\underline{q}^{M}$, we obtain the microscopic fields respectively by

$$
\underline{\nabla} u(\underline{x})=\underline{\underline{A}}(\underline{x}) \cdot \underline{\nabla} u^{M}
$$


or

$$
\underline{q}(\underline{x})=\underline{\underline{B}}(\underline{x}) \cdot \underline{q}^{M}
$$

where $\underline{\underline{A}}$ and $\underline{\underline{B}}$ are called localization tensors. Once we have obtained the microscopic fields through equations (4) (resp. (5)) for a given $\underline{\nabla} u^{M}$ (resp. $q^{M}$ ), we can deduce the other macroscopic field $q^{M}$ (resp. $\underline{\nabla} u^{M}$ ) by using the constitutive relation and a spatial averaging. We finally define the effective (homogenized) conductivity tensor $\underline{\underline{K}}_{h}$ such that

$$
\underline{q}^{M}=-\underline{\underline{K}}_{h} \cdot \underline{\nabla} u^{M}
$$

\subsection{Boundary conditions}

The homogenized conductivity tensor depends on the localization process which is governed by the choice of the boundary conditions. These boundary conditions are expressed as a function of a macroscopic field which is the input data of the microscopic problem. Classically, three sets of boundary conditions are considered [22-24, 49]. They are described below.

\subsubsection{Natural Boundary Conditions (NBC)}

Boundary value problem (3) is considered with the following Neumann boundary conditions:

$$
\underline{q}(\underline{x}) \cdot \underline{n}=\underline{q}^{M} \cdot \underline{n} \quad \text { on } \partial \Omega
$$

with $\underline{q}^{M}$ given and $\underline{n}$ the outer pointing normal of $\partial \Omega$. The solution $u$ is a priori defined up to a constant. $\bar{A}$ well-posed problem can be obtained by introducing the following solution space

$$
H_{m}^{1}(\Omega)=\left\{v \in H^{1}(\Omega) ; \int_{\Omega} v d \Omega=0\right\}
$$

The weak formulation of the problem is then:

$$
\begin{aligned}
& \text { Find } u \in H_{m}^{1}(\Omega) \text { such that } \\
& \forall \delta u \in H_{m}^{1}(\Omega), \quad \int_{\Omega} \underline{\nabla} \delta u \cdot \underline{\underline{K}} \cdot \underline{\nabla} u d \Omega=-\int_{\partial \Omega} \delta u \underline{q}^{M} \cdot \underline{n} d \Gamma
\end{aligned}
$$

Using equation (5), we obtain

$$
\underline{\nabla} u^{M}=<\underline{\nabla} u>=<-\underline{\underline{K}}^{-1} \cdot \underline{q}>=-<\underline{\underline{K}}^{-1} \cdot \underline{\underline{B}}>\cdot \underline{q}^{M}:=-\left(\underline{\underline{K}}_{h}^{n b c}\right)^{-1} \cdot \underline{q}^{M}
$$

Then, the solutions obtained from 3 different values of $\underline{q}^{M}$ (e.g. $(1,0,0)^{T},(0,1,0)^{T}$ and $\left.(0,0,1)^{T}\right)$ yield the complete characterization of $\underline{\underline{K}}_{h}^{n b c}$.

\subsubsection{Periodic Boundary Conditions (PBC)}

In this case, problem (3) is considered with the following periodic boundary conditions:

$$
\begin{array}{r}
u(\underline{x})-\underline{\nabla} u^{M} \cdot \underline{x} \text { is } \Omega \text {-periodic } \\
\text { and } \underline{q}(\underline{x}) \cdot \underline{n} \text { is } \Omega \text {-antiperiodic } \\
5
\end{array}
$$


with $\underline{\nabla} u^{M}$ given. We denote by $\tilde{u}(\underline{x})=u(\underline{x})-\underline{\nabla} u^{M} \cdot \underline{x}$. The solution $\tilde{u}$ is a priori defined up to a constant. A well-posed problem can be obtained by introducing the following solution space

$$
H_{p e r, m}^{1}(\Omega)=\left\{v \in H_{p e r}^{1}(\Omega) ; \int_{\Omega} v d \Omega=0\right\}
$$

where $H_{\text {per }}^{1}(\Omega)$ is the subspace of periodic functions in $H^{1}(\Omega)$. The weak formulation of the problem is then:

Find $\tilde{u} \in H_{p e r, m}^{1}(\Omega)$ such that

$$
\forall \delta u \in H_{p e r, m}^{1}(\Omega), \quad \int_{\Omega} \underline{\nabla} \delta u \cdot \underline{\underline{K}} \cdot \underline{\nabla} \tilde{u} d \Omega=-\int_{\Omega} \underline{\nabla} \delta u \cdot \underline{\underline{K}} \cdot \underline{\nabla} u^{M} d \Omega
$$

Thanks to the linearity of the problem, $\tilde{u}$ can be written as $\tilde{u}=\chi \cdot \underline{\nabla} u^{M}$ which yields $\underline{\nabla} u=\underline{\nabla} u^{M}+(\underline{\underline{\nabla}} \underline{\chi})^{T} \cdot \underline{\nabla} u^{M}=\left(\underline{\underline{I}}+(\underline{\underline{\nabla}} \underline{\chi})^{T}\right) \cdot \underline{\nabla} u^{M}$. Thus, the localization tensor defined in equation (4) is found to be $\underline{\underline{A}}=\underline{\underline{I}}+(\underline{\underline{\nabla}} \underline{\chi})^{T}$. We thus obtain

$$
\underline{q}^{M}=<\underline{q}>=<-\underline{\underline{K}} \cdot \underline{\nabla} u>=-<\underline{\underline{K}} \cdot\left(\underline{\underline{I}}+\underline{\underline{\nabla}}(\underline{\chi})^{T}\right)>\cdot \underline{\nabla} u^{M}:=-\underline{\underline{K}}_{h}^{p b c} \cdot \underline{\nabla} u^{M}
$$

3 different problems have to be solved for obtaining $\underline{\underline{K}}_{h}^{p b c}$ (e.g. with $\underline{\nabla} u^{M}=(1,0,0)^{T}$, $(0,1,0)^{T}$ and $\left.(0,0,1)^{T}\right)$. It can be noticed that for a material with a periodic microstructure, the localization problem defined by (3) and boundary conditions (11) can be rigorously justified by the asymptotic expansion method $[5,33,50]$. However, note that periodic boundary conditions can be used even if the microstructure is not periodic (see e.g. $[23,24])$.

\subsubsection{Essential Boundary Conditions (EBC)}

In this case, problem (3) is considered with the following Dirichlet boundary conditions:

$$
u(\underline{x})=\underline{\nabla} u^{M} \cdot \underline{x} \quad \text { on } \partial \Omega
$$

with $\underline{\nabla} u^{M}$ given. Note that the solution $u$ can be expressed under the form

$$
u(\underline{x})=\underline{\nabla} u^{M} \cdot \underline{x}+\tilde{u}(\underline{x})
$$

where $\tilde{u}$ is the solution of the following weak formulation:

Find $\tilde{u} \in H_{0}^{1}(\Omega)$ such that

$$
\forall \delta u \in H_{0}^{1}(\Omega), \quad \int_{\Omega} \underline{\nabla} \delta u \cdot \underline{\underline{K}} \cdot \underline{\nabla} \tilde{u} d \Omega=-\int_{\Omega} \underline{\nabla} \delta u \cdot \underline{\underline{K}} \cdot \underline{\nabla} u^{M} d \Omega
$$

By linearity of the problem, 3 different choices for $\underline{\nabla} u^{M}$ (e.g. $(1,0,0)^{T},(0,1,0)^{T}$ and $\left.(0,0,1)^{T}\right)$ are required to completely characterize the localization tensor $\underline{\underline{A}}$ defined in equation (4). The homogenized tensor $\underline{\underline{K}}_{h}^{e b c}$ is then obtained by

$$
6.2 e-3 \underline{q}^{M}=<\underline{q}>=<-\underline{\underline{K}} \cdot \underline{\nabla} u>=-<\underline{\underline{K}} \cdot \underline{\underline{A}}>\cdot \underline{\nabla} u^{M}:=-\underline{\underline{K}}_{h}^{e b c} \cdot \underline{\nabla} u^{M}
$$




\subsection{Unified formulation}

Weak formulations (9), (13) and (17) can be unified with an abuse of notation for EBC and $\mathrm{PBC}$, for which $\tilde{u}$ is replaced by $u$ :

Find $u \in \mathcal{V}$ such that

$\forall \delta u \in \mathcal{V}, a(\delta u, u)=l(\delta u)$

with

$$
a(\delta u, u)=\int_{\Omega} \underline{\nabla} \delta u \cdot \underline{\underline{K}} \cdot \underline{\nabla} u d \Omega
$$

and

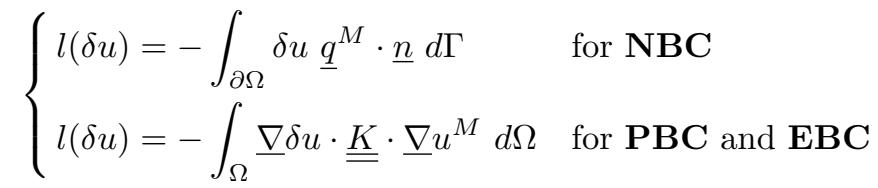

Function space $\mathcal{V}$ is defined by:

$$
\begin{cases}\mathcal{V}=H_{m}^{1}(\Omega) & \text { for } \mathbf{N B C} \\ \mathcal{V}=H_{p e r, m}^{1}(\Omega) & \text { for } \mathbf{P B C} \\ \mathcal{V}=H_{0}^{1}(\Omega) & \text { for } \mathbf{E B C}\end{cases}
$$

The generic weak formulation (19) will be used in order to simplify the presentation of the proposed solution strategy. We will then come back to the underlying problems for detailing some technical issues and for discussing some specificities of the localization problems.

\section{Tensor spaces and separated representations}

In this section, we consider a scalar field of interest $w: \Omega \rightarrow \mathbb{R}$ which can be the gray intensity level of the image representing the heterogeneous material, a component of the conductivity field $\underline{K}$ or the solution of localization problem (19). The domain $\Omega \subset \mathbb{R}^{d}$ being an image, it has a product structure. We have $\Omega=\Omega^{x} \times \Omega^{y} \times \Omega^{z}$ for $d=3$ (resp. $\Omega=\Omega^{x} \times \Omega^{y}$ for $\left.d=2\right)$, with $\Omega^{\alpha}=\left(0, l^{\alpha}\right)$. In this section, and without loss of generality, we only consider the 3-dimensional case but the different notions extend naturally to arbitrary dimension $d$. Under some regularity assumptions, a function $w$ defined on such a cartesian domain can be identified with an element of a suitable tensor space. In this section, we introduce some general notions about tensors (as elements of tensor product spaces) and their approximation using separated representations of the form:

$$
w(x, y, z) \approx w_{m}(x, y, z)=\sum_{i=1}^{m} v_{i}^{x}(x) v_{i}^{y}(y) v_{i}^{z}(z)
$$




\subsection{Tensor spaces}

For a general introduction to tensor spaces and tensor approximations, the reader can refer to Hackbusch [18]. We suppose that $w \in \mathcal{V}$ where $\mathcal{V}$ is a Hilbert tensor space defined in the set of functions $\mathbb{R}^{\Omega}$. The inner product of $\mathcal{V}$ is denoted $\langle\cdot, \cdot\rangle$ and its associated norm $\|\cdot\|$. With $\mathcal{V}^{x}\left(\right.$ resp. $\left.\mathcal{V}^{y}, \mathcal{V}^{z}\right)$ a Hilbert space of functions of $\mathbb{R}^{\Omega^{x}}$ (resp. $\mathbb{R}^{\Omega^{y}}, \mathbb{R}^{\Omega^{z}}$ ), the tensor product $\otimes$ between functions is defined in a usual way, such that

$$
\left(v^{x} \otimes v^{y} \otimes v^{z}\right)(x, y, z)=v^{x}(x) v^{y}(y) v^{z}(z)
$$

for all $v^{\alpha} \in \mathcal{V}^{\alpha}, \alpha \in\{x, y, z\}$. We define the set of rank-1 (or elementary) tensors

$$
\mathcal{R}_{1}=\left\{v=v^{x} \otimes v^{y} \otimes v^{z} ; v^{\alpha} \in \mathcal{V}^{\alpha}, \alpha \in\{x, y, z\}\right\},
$$

The algebraic tensor product $\otimes_{a}$ of spaces $\mathcal{V}^{x}, \mathcal{V}^{y}$ and $\mathcal{V}^{z}$ is then defined by

$$
\mathcal{V}^{x} \otimes_{a} \mathcal{V}^{y} \otimes_{a} \mathcal{V}^{z}=\operatorname{span}\left(\mathcal{R}_{1}\right)
$$

Finally, the Hilbert tensor space is defined by

$$
\mathcal{V}=\mathcal{V}^{x} \otimes \mathcal{V}^{y} \otimes \mathcal{V}^{z}=\overline{\mathcal{V}^{x} \otimes_{a} \mathcal{V}^{y} \otimes_{a} \mathcal{V}^{z}}\|\cdot\|
$$

where $\overline{(\cdot)}\|\cdot\|$ denotes the completion with respect to the norm $\|\cdot\|$.

In the case of a cartesian domain $\Omega=\Omega^{x} \times \Omega^{y} \times \Omega^{z}$, tensor spaces $\mathcal{V}$ that are involved in the formulation of localization problems (19), and given in (21), have the following tensor product structure:

$$
\mathcal{V}=\overline{\mathcal{V}^{x} \otimes_{a} \mathcal{V}^{y} \otimes_{a} \mathcal{V}^{z}}\|\cdot\|_{H^{1}(\Omega)}
$$

with

$$
\left\{\begin{array}{lll}
\mathcal{V}=H_{m}^{1}(\Omega), & \mathcal{V}^{\alpha}=H_{m}^{1}\left(\Omega^{\alpha}\right) & \text { for NBC } \\
\mathcal{V}=H_{p e r, m}^{1}(\Omega), & \mathcal{V}^{\alpha}=H_{p e r}^{1}, m \\
\mathcal{V}=H_{0}^{1}(\Omega), & \mathcal{V}^{\alpha}=H_{0}^{1}\left(\Omega^{\alpha}\right) & \text { for PBC } \\
& \text { for EBC }
\end{array}\right.
$$

This justifies the existence of a separated representation of type (22) for the solution of homogenization problems (19).

\subsection{Tensor decompositions}

We introduce here two classical tensor decomposition formats.

Rank-m (canonical) decomposition. For a given $m \in \mathbb{N}$, the set of rank- $m$ tensors $\mathcal{R}_{m}$ is defined by

$$
\mathcal{R}_{m}=\left\{w_{m}=\sum_{i=1}^{m} v_{i} ; v_{i} \in \mathcal{R}_{1}\right\}
$$

Definition (26) implies that for all $w \in \mathcal{V}$, there exists a sequence of tensors, $\left(w_{m}\right)_{m \in \mathbb{N}}$, such that $w_{m} \in \mathcal{R}_{m}$ and $w_{m}$ converges to $w$. Therefore, for any $w \in \mathcal{V}$, this condition justifies the existence of the approximation of type (22) with an arbitrary accuracy. 
Tucker tensors. For a given multi-index $\mathbf{r}=\left(r^{x}, r^{y}, r^{z}\right) \in \mathbb{N}^{3}$, we define the Tucker tensors set $\mathcal{T}_{\mathbf{r}}$ as follows:

$$
\mathcal{T}_{\mathbf{r}}=\left\{w_{\mathbf{r}}=\sum_{i=1}^{r^{x}} \sum_{j=1}^{r^{y}} \sum_{k=1}^{r^{z}} \lambda_{i j k} v_{i}^{x} \otimes v_{j}^{y} \otimes v_{k}^{z} ; \lambda_{i j k} \in \mathbb{R}, v_{p}^{\alpha} \in \mathcal{V}^{\alpha},<v_{p}^{\alpha}, v_{q}^{\alpha}>_{\alpha}=\delta_{p q}\right\}
$$

with $\langle\cdot, \cdot\rangle_{\alpha}$ the inner product on Hilbert space $\mathcal{V}^{\alpha}$. An approximation $w_{\mathbf{r}} \in \mathcal{T}_{\mathbf{r}}$ of an element $w \in \mathcal{V}$ is called a rank-r Tucker approximation of $w$. We have the property that $\mathcal{R}_{r} \subset \mathcal{T}_{(r, r, r)}$. Therefore, for all $w \in \mathcal{V}$, there also exists a sequence $\left(w_{m}\right)_{m \in \mathbb{N}}$, with $w_{m} \in \mathcal{T}_{\mathbf{r}_{m}}$ and such that $w_{m}$ converges to $w$. The reader can refer to [27] for a review of definitions and constructions of tensor representations in finite dimensional algebraic tensor spaces $\mathcal{V}=\mathbb{R}^{N_{x}} \otimes \mathbb{R}^{N_{y}} \otimes \mathbb{R}^{N_{z}}$.

\section{Proper Generalized Decomposition}

The homogenization problem we want to solve has been recasted as follows (see section $1.3)$ :

$$
\begin{aligned}
& \text { Find } u \in \mathcal{V} \text { such that } \\
& \forall \delta u \in \mathcal{V}, a(\delta u, u)=l(\delta u)
\end{aligned}
$$

where $\mathcal{V}$ is a tensor Hilbert space with tensor structure given in (28). Proper Generalized Decomposition (PGD) methods constitute a family of algorithms for the construction of a tensor approximation of the solution $u$ of (31), without a priori information on the solution. It can be achieved by formulating best approximation problems on tensors subsets using operator-based norms instead of natural norms in tensor space $\mathcal{V}$. In this section, we first recall the principle of PGD methods. We then introduce a now classical algorithm for the construction of rank- $m$ approximations and we recall some properties of this approximation. Then, we will introduce a new algorithm for the progressive construction of a Tucker representation of the solution. This algorithm provides better convergence properties than classical PGD definitions based on canonical decompositions.

Remark 1. In this section, we consider that the problems are formulated on a $d$ dimensional domain $\Omega \subset \mathbb{R}^{d}$, with $\Omega=\Omega^{x} \times \Omega^{y} \times \Omega^{z}$ for $d=3$ or $\Omega=\Omega^{x} \times \Omega^{y}$ for $d=2$. The notation $\otimes_{\alpha}$ will stand for $\otimes_{\alpha \in\{x, y, z\}}$ for $d=3$ and $\otimes_{\alpha \in\{x, y\}}$ for $d=2$.

\subsection{A priori definition of an approximation on a tensor subset}

Let us consider a tensor subset $\mathcal{S}$ (e.g. rank-1 tensors set, Tucker tensors set...). Given a norm $\|\cdot\|$ in $\mathcal{V}$, a best approximation $u^{*} \in \mathcal{S}$ of $u \in \mathcal{V}$ can be naturally defined as

$$
\left\|u-u^{*}\right\|=\min _{v \in \mathcal{S}}\|u-v\|
$$

The idea is to find an approximation $u^{*}$ of $u$ without a priori information on $u$. Therefore, the norm must be chosen in such a way that problem (32) can be solved without knowing $u$. In the present application, where $u$ is solution of (31) with $a$ a symmetric coercive and continuous bilinear form, the norm $\|\cdot\|$ and associated inner product $<\cdot, \cdot\rangle$ can be chosen as follows:

$$
\|v\|^{2}=a(v, v), \quad<u, v>=a(u, v)
$$


where the norm $\|\cdot\|$ is equivalent to the initial norm on $\mathcal{V}$. We then have

$$
\|u-v\|^{2}=a(u, u)+a(v, v)-2 a(u, v)=a(u, u)+a(v, v)-2 l(v),
$$

and minimization problem (32) is then equivalent to

$$
J\left(u^{*}\right)=\min _{v \in \mathcal{S}} J(v), \quad J(v)=\frac{1}{2} a(v, v)-l(v)
$$

We note that problem (33) that defines the tensor approximation in $\mathcal{S}$ does not involve the solution $u$. It makes the approximation $u^{*}$ computable without a priori information on $u$. Of course, tensor subsets $\mathcal{S}$ must be such that minimization problems on $\mathcal{S}$ are wellposed. Moreover, tensor subsets $\mathcal{S}$ (such as rank-1 tensors, rank-r Tucker tensors) are manifolds which are not necessarily linear spaces, so that even if problem (31) is a linear approximation problem, minimization problem (33) is no more a linear approximation problem and specific algorithms have to be devised.

Remark 2. Note that a necessary condition for optimality of $u^{*}$ writes

$$
a\left(u^{*}, \delta v\right)=l(\delta v) \quad \forall \delta v \in T_{u^{*}}(\mathcal{S})
$$

where $T_{u^{*}}(\mathcal{S})$ is the tangent linear space to $\mathcal{S}$ at $u^{*}$.

\subsection{Construction of a rank-m (canonical) decomposition}

The aim is to find an optimal rank- $m$ approximation $u_{m} \in \mathcal{R}_{m}$ of $u$ of the form

$$
u_{m}=\sum_{i=1}^{m} v_{i}
$$

with $v_{i} \in \mathcal{R}_{1}$. A direct definition of an optimal approximation in $\mathcal{R}_{m}$ would be defined by the optimization problem (32). However, it is well known that for $m \geq 2$ and $d \geq 3$, $\mathcal{R}_{m}$ is not a weakly closed set so that the minimization problem (32) is ill-posed for $\mathcal{S}=\mathcal{R}_{m}$ [15]. A modified direct definition of an approximation with arbitrary precision $\varepsilon>0$ could be formulated as follows:

Find $u_{m}$ such that

$$
\left\|u-u_{m}\right\| \leq \inf _{v \in \mathcal{R}_{m}}\|u-v\|+\varepsilon
$$

The solution of this problem yields the whole set of rank-1 elements $\left\{v_{i}\right\}_{i=1}^{m}$ at once. In order to avoid the introduction of the tolerance $\varepsilon$ (i.e. $\varepsilon=0$ ), it could be possible to consider a weakly closed subset of $\mathcal{R}_{m}$ (e.g. by adding some orthogonality constraints between rank-one elements). However, these direct constructions are computationnally expensive since when increasing $m$, optimization problems are defined on sets with increasing dimensionality and computational complexity drastically increases.

In order to circumvent the above difficulties, a progressive definition of PGD is classically introduced $[10,15]$, which consists in building the sequence $\left(v_{i}\right)_{1 \leq i \leq m}$ term by term (in a greedy fashion). Even if the progressive definition is sub-optimal compared to the direct PGD approach, $\mathcal{R}_{1}$ is weakly closed [15] so that successive best approximation problems on $\mathcal{R}_{1}$ are well-posed. Moreover, optimization problems on $\mathcal{R}_{1}$ have 


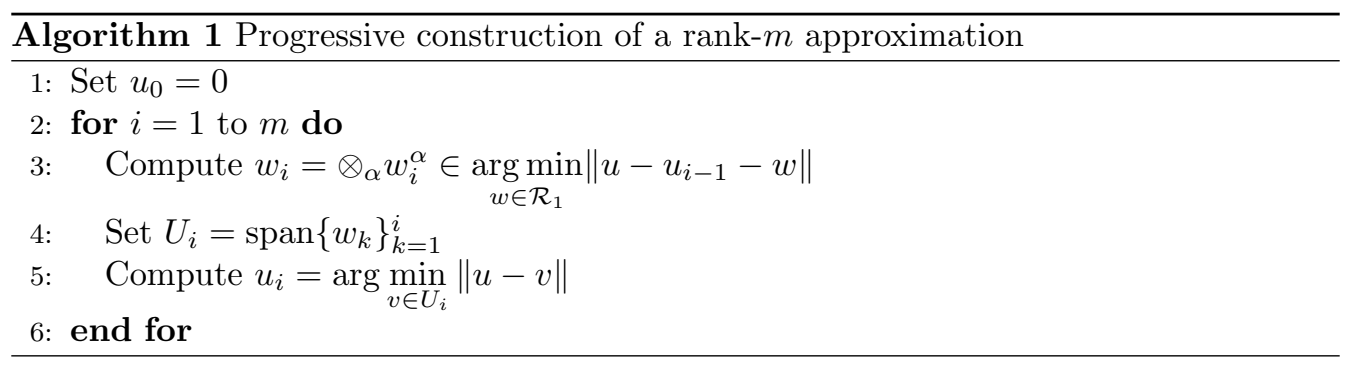

almost the same complexity, so that the complexity of computing a rank- $m$ approximation scales (approximately) linearly with the rank $m$. The algorithm used is defined in Algorithm 1. Step 5 of Algorithm 1 corresponds to a classical projection onto the subspace spanned by all rank-one elements previously generated. At step $m$, the solution $u_{m}=\arg \min _{v \in U_{m}}\|u-v\|$ can be written under the form

$$
u_{m}=\sum_{i=1}^{m} \lambda_{i} w_{i} \quad \text { with } w_{i}=\otimes_{\alpha} w_{i}^{\alpha}
$$

where the $\left(\lambda_{i}\right)_{i=1}^{m} \in \mathbb{R}^{m}$ are coefficients that are solutions of the following system of $m$ equations:

$$
\sum_{j=1}^{m} A_{i j} \lambda_{j}=b_{i}, \quad \forall i \in\{1, \ldots, m\}
$$

with

$$
A_{i j}=a\left(w_{i}, w_{j}\right)=a\left(\otimes_{\alpha} v_{i}^{\alpha}, \otimes_{\alpha} v_{j}^{\alpha}\right), \quad b_{i}=l\left(w_{i}\right)=l\left(\otimes_{\alpha} v_{i}^{\alpha}\right)
$$

Different algorithms have been proposed for computing $w_{m} \in \arg \min _{v \in \mathcal{R}_{1}} \| u-u_{m-1}-$ $v \|$ (step 3). One possibility is an alternating minimization algorithm presented in $\mathrm{Al}-$ gorithm 2. The integer $k_{\max }$ represents the maximum number of iterations and the tolerance $\varepsilon_{s}$ is associated to a stagnation criterium. In practice, we will take $k_{\max }=10$ and $\varepsilon_{s}=5.10^{-2}$.

Remark 3. In the case where $\|\cdot\|$ is the classical canonical norm on a finite dimensional space, Algorithm 2 corresponds to the Alternating Least Squares (ALS) algorithm on $\mathcal{R}_{1}$ [8, 20, 27].

Remark 4. If the domain $\Omega$ is 2-dimensional, the method can be considered as a generalization of the SVD with respect to general norms on Hilbert spaces [15].

Minimization problem of step 5 of Algorithm 2, corresponding to the computation of $v^{\alpha}$, is equivalent to

$$
\min _{v^{\alpha} \in \mathcal{V}^{\alpha}} \frac{1}{2} a\left(\otimes_{\beta} v^{\beta}, \otimes_{\beta} v^{\beta}\right)+a\left(u_{m-1}, \otimes_{\beta} v^{\beta}\right)-l\left(\otimes_{\beta} v^{\beta}\right)
$$




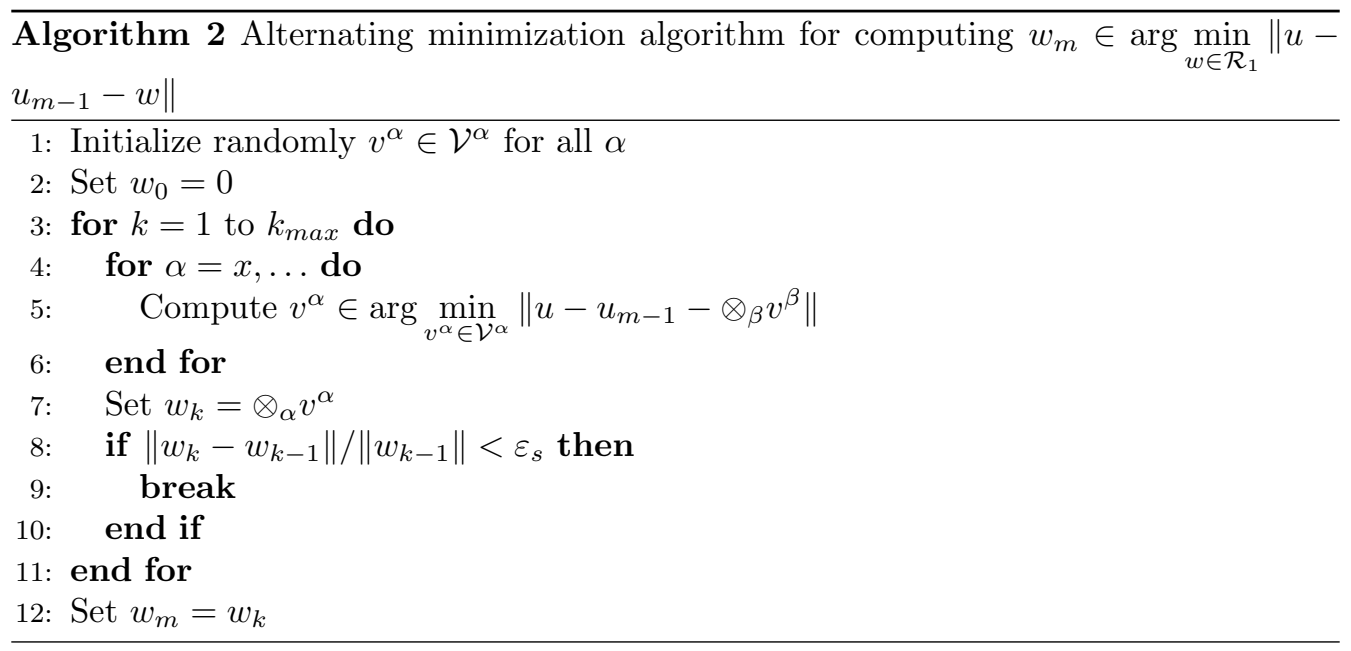

It reduces to the solution of the following linear problem:

$$
\begin{aligned}
& \text { Find } v^{\alpha} \in \mathcal{V}^{\alpha} \text { such that } \\
& \forall \delta \varphi \in \mathcal{V}^{\alpha}, a^{\alpha}\left(\delta \varphi, v^{\alpha}\right)=l_{m}^{\alpha}(\delta \varphi)
\end{aligned}
$$

with

$$
\begin{aligned}
& a^{\alpha}(\delta \varphi, \varphi)=a\left(\delta \varphi \otimes\left(\otimes_{\beta \neq \alpha} v^{\beta}\right), \varphi \otimes\left(\otimes_{\beta \neq \alpha} v^{\beta}\right)\right) \\
& l_{m}^{\alpha}(\delta \varphi)=l\left(\delta \varphi \otimes\left(\otimes_{\beta \neq \alpha} v^{\beta}\right)\right)-a\left(\delta \varphi \otimes\left(\otimes_{\beta \neq \alpha} v^{\beta}\right), u_{m-1}\right)
\end{aligned}
$$

Problem (39) is a one-dimensional problem defined on $\mathcal{V}^{\alpha} \subset H^{1}\left(\Omega^{\alpha}\right)$. The proposed algorithm then only involves the solution of uncoupled one-dimensional problems, which is the reason for the efficiency of the PGD method.

Remark 5. Assuming that $\mathcal{V}^{\alpha}=\mathbb{R}^{n}, \forall \alpha$, and that the complexity of a linear solver on a problem of size $n$ is $n^{3}$, then the construction of all the vectors of a rank-r decomposition with algorithm 2 requires at most $r k_{\text {max }} d n^{3}$ operations. The update of the $\lambda$ 's requires $1+2^{3}+\ldots+r^{3}=\frac{1}{4} r^{2}(r+1)^{2}$ operations. Finally the complexity of the algorithm 1 is $\frac{1}{4} r^{2}(r+1)^{2}+r k_{\max } d n^{3}$.

\subsection{Construction of a rank-r Tucker decomposition}

An approximation of the solution using a Tucker format could be searched by solving an optimization problem:

$$
\left\|u-u_{\mathbf{r}}\right\|=\min _{v \in \mathcal{T}_{\mathbf{r}}}\|u-v\|
$$

This problem is well-posed [14]. However, this direct construction of a Tucker approximation $u_{\mathbf{r}} \in \mathcal{T}_{\mathbf{r}}$ is computationally expensive and when the objective is to find an approximation with a desired accuracy, we will rather prefer an adaptive construction.

We now propose a sub-optimal but progressive construction of a sequence of Tucker approximations $\left\{u_{m}\right\}_{m \in \mathbb{N}}$ of the solution, where $u_{m}$ is the best approximation of the 
solution in a linear subspace $U_{m}$ of $\mathcal{T}_{(m, \ldots, m)}$, with $U_{m}$ of the form:

$$
U_{m}=\otimes_{\alpha} U_{m}^{\alpha}
$$

that means $U_{m}=U_{m}^{x} \otimes U_{m}^{y} \otimes U_{m}^{z}$ for dimension $d=3$ or $U_{m}^{x} \otimes U_{m}^{y}$ for dimension $d=2$. Linear subspaces $U_{m}^{\alpha} \subset \mathcal{V}^{\alpha}$ are defined progressively, by completing the previous spaces $U_{m-1}^{\alpha}$ by fonctions $w_{m}^{\alpha}$ extracted from a rank-one corrections $w_{m}=\otimes_{\alpha} w_{m}^{\alpha}$ of $u_{m-1}$. The sequences of linear spaces $\left\{U_{m}^{\alpha}\right\}_{m \in \mathbb{N}}$ (and $\left\{U_{m}\right\}_{m \in \mathbb{N}}$ ) are increasing with $m$ (with respect to inclusion). Let us detail this procedure.

Let $U_{0}^{\alpha}=0$ and $U_{0}=0$. At iteration $m$, knowing $u_{m-1} \in U_{m-1}=\otimes_{\alpha} U_{m-1}^{\alpha}$, we start by computing an optimal correction $w_{m} \in \mathcal{R}_{1}$ of $u_{m-1}$, defined by

$$
w_{m}=\otimes_{\alpha} w_{m}^{\alpha} \in \underset{w \in \mathcal{R}_{1}}{\operatorname{argmin}}\left\|u-u_{m-1}-w\right\|
$$

The new linear subspace $U_{m}^{\alpha}$ is then defined by

$$
U_{m}^{\alpha}=U_{m-1}^{\alpha}+\operatorname{span}\left\{w_{m}^{\alpha}\right\}
$$

which has a dimension $\operatorname{dim}\left(U_{m}^{\alpha}\right):=r_{m}^{\alpha} \leq m$. The linear space $U_{m}$ is then defined by (41) and has a dimension $\operatorname{dim}\left(U_{m}\right):=r_{m}=\prod_{\alpha} r_{m}^{\alpha}$. The next approximation $u_{m} \in U_{m}$ is then defined by the following linear approximation problem:

$$
\left\|u-u_{m}\right\|=\min _{v \in U_{m}}\|u-v\|
$$

This procedure requires the solution of a succession of best approximation problems (42) on the manifold $\mathcal{R}_{1}$, which are nonlinear approximation problems with moderate complexity, and a succession of best approximation problems on linear subspaces $U_{m}$, which are approximation problems with increasing complexity $r_{m}$. However, these latter problems remain classical linear approximation problems. The algorithm is summed up in Algorithm 3. Step 6 (best approximation in $\mathcal{R}_{1}$ ) can be accomplished thanks to the

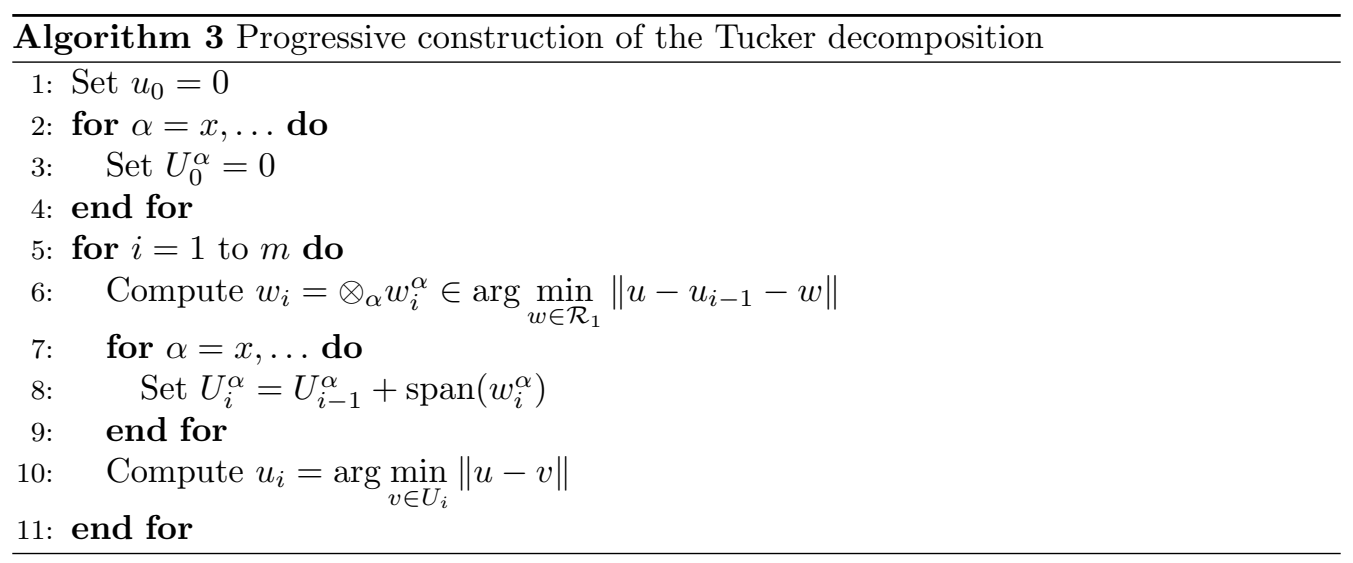

alternating minimization algorithm (Algorithm 2). In practice, an orthonormal basis 
$\left\{v_{j}^{\alpha}\right\}_{1 \leq j \leq r_{m}^{\alpha}}$ of $U_{m}^{\alpha}$ is built using the Gram-Schmidt procedure applied to the generating set $\left\{w_{j}^{\alpha}\right\}_{1 \leq j \leq m}$. The approximation $u_{m}$, solution of (43), can be written under the form

$$
u_{m}=\sum_{i \in \mathcal{I}_{m}} \lambda_{i} v_{i}, \quad v_{i}=\otimes_{\alpha} v_{i_{\alpha}}^{\alpha}
$$

with $\mathcal{I}_{m}=\left\{\boldsymbol{i} \in \mathbb{N}^{d} ; 1 \leq i_{\alpha} \leq r_{m}^{\alpha}\right\}$. The set of coefficients $\left(\lambda_{\boldsymbol{i}}\right)_{\boldsymbol{i} \in \mathcal{I}_{m}}$ is solution of a linear system of equations

$$
\sum_{j \in \mathcal{I}_{m}} A_{i j} \lambda_{j}=b_{i}, \quad i \in \mathcal{I}_{m}
$$

with

$$
A_{i j}=a\left(v_{\boldsymbol{i}}, v_{\boldsymbol{j}}\right)=a\left(\otimes_{\alpha} v_{i_{\alpha}}^{\alpha}, \otimes_{\alpha} v_{j_{\alpha}}^{\alpha}\right), \quad b_{\boldsymbol{i}}=l\left(v_{\boldsymbol{i}}\right)=l\left(\otimes_{\alpha} v_{i_{\alpha}}^{\alpha}\right)
$$

Remark 6. The orthonormalization of basis functions allows to detect if at step $m$, a new function $w_{m}^{\alpha}$ is contained in the previous linear space $U_{m-1}^{\alpha}$, in which case $U_{m}^{\alpha}=U_{m-1}^{\alpha}$ and $r_{m}^{\alpha}=r_{m-1}^{\alpha}$. This allows to ensure the uniqueness of the best approximation $u_{m}$ in $U_{m}$ (regularity of system (44)). Also, it allows to automatically detect and take part of the independence (or quasi-independence) of the solution with respect to a certain variable.

Remark 7. Again, assuming that $\mathcal{V}^{\alpha}=\mathbb{R}^{n}, \forall \alpha$, and that the complexity of a linear solver on a problem of size $n$ is $n^{3}$, then the construction of all the vectors of a rank$\left(r_{1}, \ldots, r_{d}\right)$ decomposition requires at most $r k_{\max } d n^{3}$ operations, for $r_{1}=\ldots=r_{d}=r$. The update of the core tensor $\alpha$ requires at most $1+2^{3 d}+\ldots+r^{3 d} \leq r^{3 d+1}$ operations. Finally, the complexity of the algorithm 3 is bounded by $r^{3 d+1}+r k_{\max } d n^{3}$. This indicates that we should use this method in $2 D$ or when the geometry presents particular symmetries such that one or two $r_{\alpha}$ are small compared to the others, like in section 5.2 for instance.

\section{Tensor format for image-based homogenization problems}

In order to apply the PGD method to the solution of numerical homogenization problems, specific reformulations and approximations have to be introduced in order to recast the problem in a suitable format adapted to tensor-based methods. These specific treatments concern the separated representation of the conductivity field $\underline{\underline{K}}$, yielding an approximation of operator under a tensor format, and the introduction of suitable reformulations for imposing the different types of boundary conditions.

In $[30,31]$, the authors already introduced a similar computational method for homogenization in the EBC case and for simple geometries. In the present contribution, these works are extended to the different boundary conditions that are classically used in computational homogenization, which necessitates the introduction of suitable reformulations of the variational problem. Moreover, we introduce specific numerical treatments for dealing with real geometries extracted from images. 


\subsection{Separated representation of the conductivity field}

Let us consider a composite material with two phases numbered 1 and 2 in which the conductivity is constant and is denoted $\underline{\underline{K}}_{1}$ and $\underline{\underline{K}}_{2}$ respectively. If $I: \Omega \rightarrow\{0,1\}$ is the characteristic function of phase 1 , the local conductivity $\underline{\underline{K}}$ is rewritten

$$
\underline{\underline{K}}(\underline{x})=I(\underline{x}) \underline{\underline{K}}_{1}+(1-I(\underline{x})) \underline{\underline{K}}_{2}=\underline{\underline{K}}_{2}+I(\underline{x})\left(\underline{\underline{K}}_{1}-\underline{\underline{K}}_{2}\right)
$$

Therefore, a separated representation of the scalar field $I \in L^{2}(\Omega)=\otimes_{\alpha} L^{2}\left(\Omega^{\alpha}\right)$ yields a separated representation of $K$. Suppose that the image contains $P^{x} \times P^{y} \times P^{z}$ voxels for $d=3$, or $P^{x} \times P^{y}$ pixels for $d=2$. For $d=3$, the discrete characteristic function can be written

$$
I(x, y, z)=\sum_{i=1}^{P^{x}} \sum_{j=1}^{P^{y}} \sum_{k=1}^{P^{z}} I_{i j k} \phi_{i}^{x}(x) \phi_{j}^{y}(y) \phi_{k}^{z}(z)
$$

where $I_{i j k}$ is the value of $I$ in the voxel $(i, j, k)$ and where the $\left\{\phi_{i}^{\alpha}\right\}_{1 \leq i \leq P^{\alpha}}$ are piecewise constant interpolation functions. For $d=2$, we can write

$$
I(x, y)=\sum_{i=1}^{P^{x}} \sum_{j=1}^{P^{y}} I_{i j} \phi_{i}^{x}(x) \phi_{j}^{y}(y)
$$

where $I_{i j}$ is the value of $I$ in the pixel $(i, j)$. We then identify function $I$ with $\mathbf{I}=\left(I_{i j k}\right) \in$ $\mathbb{R}^{P^{x}} \otimes \mathbb{R}^{P^{y}} \otimes \mathbb{R}^{P^{z}}$ for $d=3$ (resp. $\mathbf{I}=\left(I_{i j}\right) \in \mathbb{R}^{P^{x}} \otimes \mathbb{R}^{P^{y}}$ for $d=2$ ).

In this section, we propose to slightly smooth the geometry in order to obtain a lower rank tensor decomposition. This can be seen as a particular approximation of the geometry. Note that voxel-based, remeshing or level-set techniques also introduce approximations which result in different representations of the actual geometry.

\subsubsection{Singular value decompositions}

We now equip $\otimes_{\alpha} \mathbb{R}^{P^{\alpha}}$ with the canonical inner product, which is defined for $\mathbf{X}, \mathbf{Y} \in$ $\otimes_{\alpha} \mathbb{R}^{P^{\alpha}}$ by

$$
\begin{array}{ll}
<\mathbf{X}, \mathbf{Y}>=\sum_{i=1}^{P^{x}} \sum_{j=1}^{P^{y}} X_{i j} \cdot Y_{i j} & \text { for } d=2 \\
<\mathbf{X}, \mathbf{Y}>=\sum_{i=1}^{P^{x}} \sum_{j=1}^{P^{y}} \sum_{k=1}^{P^{z}} X_{i j k} \cdot Y_{i j k} & \text { for } d=3
\end{array}
$$

The associate norm is denoted $\|\cdot\|$. For $d=2$, it is well known that the best rank- $m$ approximation of $\mathbf{I}$ with respect to the norm $\|\cdot\|$ is the classical Singular Value Decomposition (SVD) truncated at rank $m$. For $d=3$, several alternatives have been proposed for extending the concept of SVD. One could apply the progressive PGD approach presented in section 3, with the canonical norm $\|\cdot\|$, to obtain a separated representation of $\mathbf{I}$. However, much more efficient constructions have been proposed for tensor decomposition in $\mathbb{R}^{P^{x}} \otimes \mathbb{R}^{P^{y}} \otimes \mathbb{R}^{P^{z}}$, the tensor to be decomposed being known a priori [27]. For example, 
the ALS algorithm computes an approximation of $\mathbf{I}$ on $\mathcal{R}_{m}$ by approximating the best approximation problem on $\mathcal{R}_{m}$. We can also mention the Higher-Order Singular Value Decomposition (HOSVD) that defines an approximation of $\mathbf{I}$ in the Tucker set $\mathcal{T}_{\mathbf{r}}$. An implementation of these methods can be found in the MATLAB ${ }^{\mathrm{TM}}$ Tensor Toolbox [4]. Note that the above definitions can be considered as particular cases of PGD methods for particular choices of norm. In fact, for the above particular norm (which is a crossnorm), these methods can be considered as multidimensional versions of the SVD.

Finally, we obtain a separated approximation $\mathbf{I}_{m}$ of $\mathbf{I}$ in $\mathcal{R}_{m}$ (or eventually in $\mathcal{T}_{\mathbf{r}}$ for the Tucker format). The error $\varepsilon=\left\|\mathbf{I}-\mathbf{I}_{m}\right\| /\|\mathbf{I}\|$ can be controlled in order to provide a desired accuracy on the description of the geometry. Note that the approximation $\mathbf{I}_{m}$ can be identified with an approximation $I_{m}$ of $I$ in the tensor space $L^{2}(\Omega)$.

\subsubsection{Regularized indicator functions}

When computing a separated decomposition $I_{m}$ of $I$ using classical tensor decompositions, we observe that the number of terms needed to have a good representation of $I$ is highly dependent on the regularity properties of function $I$. The indicator function, which presents strong discontinuities at the material interfaces, is a typical example of an irregular function which yields a bad convergence of separated representations. In order to circumvent this convergence issue, the image is slightly smoothed before using tensor approximations. In practice, the iso zero of a level-set $\phi$ defined from the image is used to represent the interfaces. A smoothed indicator function $I_{s}$ is obtained by the following operation

$$
I_{s}=\frac{1}{2}\left(1+\tanh \left(\frac{2 \phi}{\delta}\right)\right)
$$

where $\delta$ represents a characteristic length. This formula is applied on the whole level-set, no matter the distance to the interfaces. The effect of smoothing is illustrated on figure 1 .

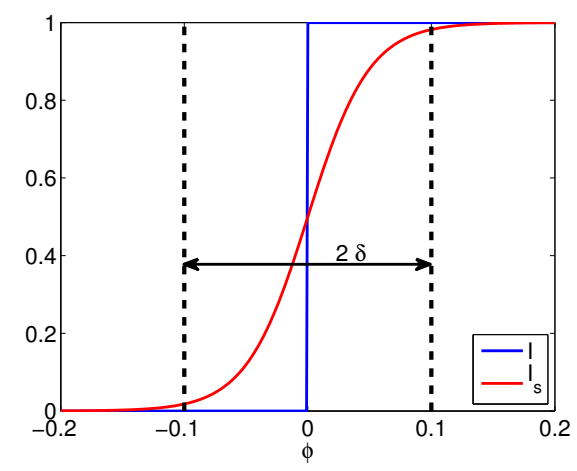

Figure 1: Smoothing effect on the characteristic function

\subsubsection{Illustration}

We here illustrate the impact of the regularization of the indicator function on a $2 \mathrm{D}$ example. We consider the $2 \mathrm{D}$ image $I$ with $512 \times 512$ pixels represented in figure 2 . A 


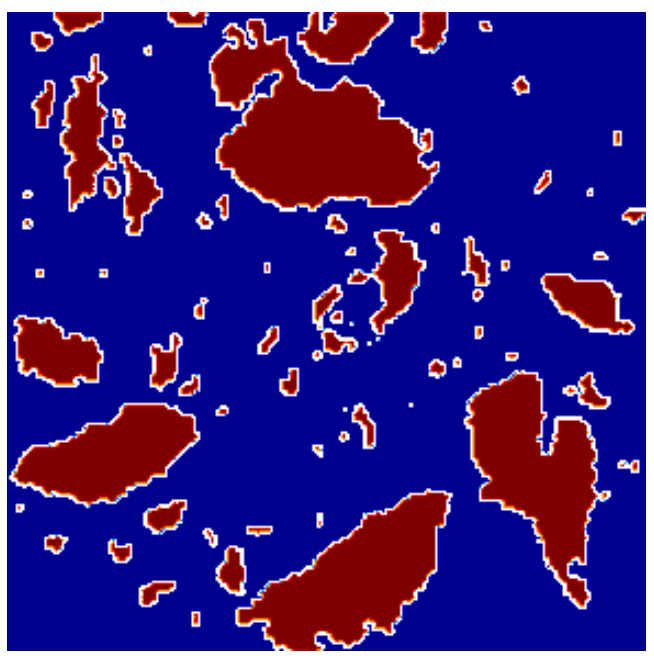

Figure 2: 2D image taken from [17]

very small smoothing is applied ( $\delta=2$ pixels). In this 2 -dimensional case, the rank- $m$ tensor approximation corresponds to a rank- $m$ truncated SVD. On figure 3 , we illustrate the convergence of SVD applied to the original image $I$ or to the smoothed image $I_{s}$. This figure shows that even for a small smoothing, we obtain a faster convergence of the decomposition. Indeed, for a fixed error $\epsilon$ of $10^{-2}$, about 100 additional modes are necessary for the decomposition of the original image. The smoothing then yields to lower

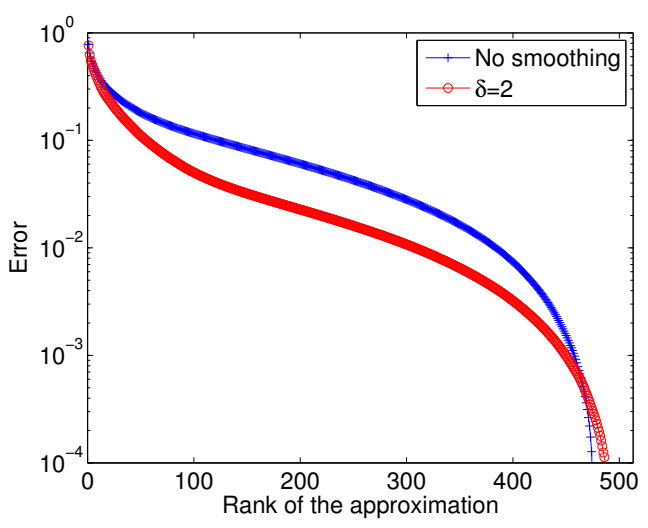

Figure 3: Convergence in $L^{2}$ norm of the separated representations of the initial and smoothed images with respect to their rank.

rank representations and therefore to more efficient tensor-based algorithms. However, the smoothing introduces an approximation of the geometry that has to be controlled. Note that another advantage of the smoothing of the characteristic function is that it removes the oscillations at the boundaries and prevents the approximation $I_{m}$ to be negative, which would lead to a negative conductivity in some regions of the domain and 
therefore to an ill-posed boundary value problem.

\subsection{Tensor format of boundary conditions}

4.2.1. Natural Boundary conditions (NBC)

Problem (9) is reformulated as follows:

$$
\begin{aligned}
& \text { Find } u \in H^{1}(\Omega) \text { such that } \forall \delta u \in H^{1}(\Omega) \\
& \int_{\Omega} \underline{\nabla} \delta u \cdot \underline{\underline{K}} \cdot \underline{\nabla} u d \Omega+\gamma \int_{\Omega} \delta u d \Omega \int_{\Omega} u d \Omega=-\int_{\partial \Omega} \delta u \underline{q}^{M} \cdot \underline{n} d \Gamma
\end{aligned}
$$

with $\gamma>0$. For any $\gamma$, the solution of (48) verifies $\int_{\Omega} u d \Omega=0$ and therefore, $u \in H_{m}^{1}(\Omega)$ is the solution of the initial problem. We solve $d$ problems associated with $\underline{\nabla} u^{M}=\underline{e}_{\alpha}$, where $\left\{e_{\alpha}\right\}$ is the canonical basis of $\mathbb{R}^{d}$. The right-hand side can be expressed as a sum over the boundary of the cartesian domain. The normal flux $\underline{q}^{M} \cdot \underline{n}$ being constant on each face, each term in the right-hand side is a rank-one tensor, so that the resulting right-hand side can be represented by rank- $2^{d}$ tensor, where $2^{d}$ is the number of boundary faces in dimension $d$.

\subsubsection{Periodic Boundary Conditions (PBC)}

Problem (13) is first reformulated as follows:

$$
\begin{aligned}
& \text { Find } \tilde{u} \in H_{p e r}^{1}(\Omega) \text { such that } \forall \delta u \in H_{p e r}^{1}(\Omega) \\
& \int_{\Omega} \underline{\nabla} \delta u \cdot \underline{\underline{K}} \cdot \underline{\nabla} \tilde{u} d \Omega+\gamma \int_{\Omega} \delta u d \Omega \int_{\Omega} u d \Omega=-\int_{\Omega} \underline{\nabla} \delta u \cdot \underline{\underline{K}} \cdot \underline{\nabla} u^{M} d \Omega
\end{aligned}
$$

with $\gamma>0$. For any $\gamma$, the solution of (49) verifies $\int_{\Omega} u d \Omega=0$ and therefore, $u \in$ $H_{\text {per }, m}^{1}(\Omega)$ is the solution of the initial problem. The term $\underline{\nabla} u^{M}$ is uniform over $\Omega$. The tensor format of the right-hand side then follows directly from the tensor format of the conductivity field. We solve $d$ problems associated with $\underline{\nabla} u^{M}=\underline{e}_{\alpha}$, where $\left\{e_{\alpha}\right\}$ is the canonical basis of $\mathbb{R}^{d}$.

The $\Omega$-periodicity of tensor approximations of type $u_{m}=\sum_{i=1}^{m} \otimes_{\alpha} v_{i}^{\alpha}$ is naturally obtained by imposing $\Omega^{\alpha}$-periodicity for all 1-dimensional functions $v_{i}^{\alpha}$.

\subsubsection{Essential Boundary conditions (EBC)}

The weak formulation of the boundary value problem associated with EBC is (17). $\underline{\nabla} u^{M}$ being uniform on $\Omega$, the tensor format of the right-hand side follows directly from the tensor format of the conductivity field. We solve $d$ problems associated with $\underline{\nabla} u^{M}=$ $\underline{e}_{\alpha}$, where $\left\{e_{\alpha}\right\}$ is the canonical basis of $\mathbb{R}^{d}$.

Finally, we impose homogeneous boundary conditions for all 1-dimensional functions $v_{i}^{\alpha} \in H_{0}^{1}\left(\Omega^{\alpha}\right)$ in tensor approximations of type $u_{m}=\sum_{i=1}^{m} \otimes_{\alpha} v_{i}^{\alpha}$, thus imposing homogeneous boundary conditions for $u_{m} \in H_{0}^{1}(\Omega)$.

\section{First applications}

\subsection{PGD using canonical tensor format}

The aim is to illustrate the behavior of the progressive PGD method and estimate the impact of PGD approximations on the quality of the quantities of interest which are the homogenized tensors. 
We consider the $2 \mathrm{D}$ image represented in figure 4 . The picture contains $128 \times 128$ pixels and is associated with a domain $\Omega=(0,1)^{2}$. The domain contains random inclusions in a matrix. The materials phases are isotropic with conductivities $k_{i}=10 \mathrm{~W} \cdot \mathrm{m}^{-1} \cdot \mathrm{K}^{-1}$ for the inclusions and $k_{m}=1 W \cdot m^{-1} \cdot K^{-1}$ for the matrix. We use a characteristic length of $\delta=1$ pixel for the smoothing of the indicator function.

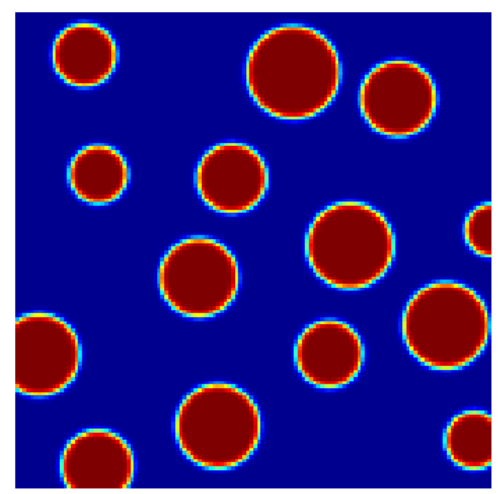

Figure 4: Random inclusions into a matrix

Problem (19) has been solved for NBC, PBC and EBC using a classical Finite Element Method (FEM). These FEM solutions and the corresponding homogenized tensors are taken as reference solutions.

In order to quantify only the error coming from the PGD approximation, we use an exact representation of the original image. This avoids any degradation of the geometry due to truncation of its decomposition. We here apply a progressive PGD algorithm to construct rank- $m$ separated representations of the solutions. On figure 5 is plotted the convergence of the PGD approximations with respect to the rank of the approximation. We observe similar convergence properties for different problems associated with the 3 types of boundary conditions. In fact, tensor decompositions being related to singular value decompositions (spectral decompositions), the observed convergence reflects the spectral content of the solutions. The observed plateaux can be explained by clusters of singular values. Figure 6 shows the convergence of the corresponding estimations of the homogenized tensors.

We can see that in the 3 cases, we have a good convergence of the homogenized tensor with the rank of the approximation. Besides, a slower convergence is observed for the NBC case. This can be explained by the fact that for the NBC case, high frequency modes in the separated approximations have non negligible spatial means, as opposed to the cases of $\mathrm{PBC}$ and $\mathrm{EBC}$.

If we increase the contrast, by taking $k_{i}=1000 W \cdot \mathrm{m}^{-1} \cdot K^{-1}$, we observe on figure 7 a slower convergence. In figure 8 , we also observe a slower convergence for the homogenized tensors. The large increase of the contrast deteriorates the conditioning of the operator. In order to improve the present results, preconditioning techniques should be introduced. Note that some preconditioning techniques have already been introduced for operators in tensor format, see e.g. $[26,32,55]$. Preconditioning techniques adapted to the present framework are under investigation and will be introduced in a subsequent paper. 


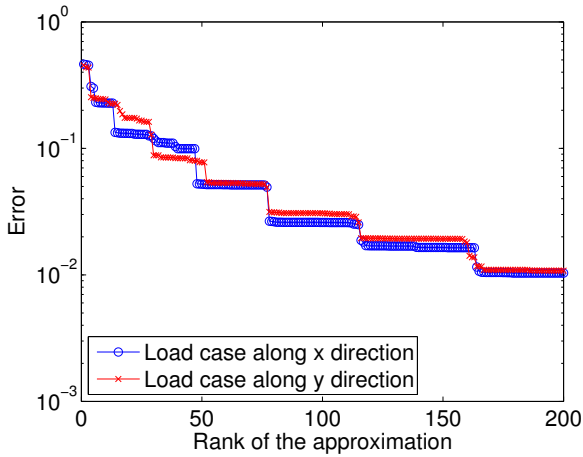

(a) NBC

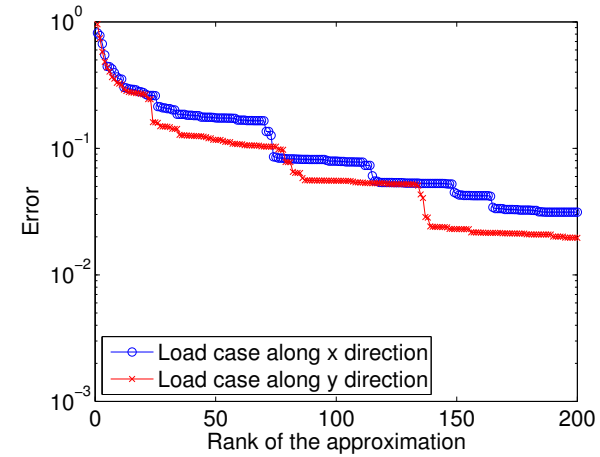

(b) PBC

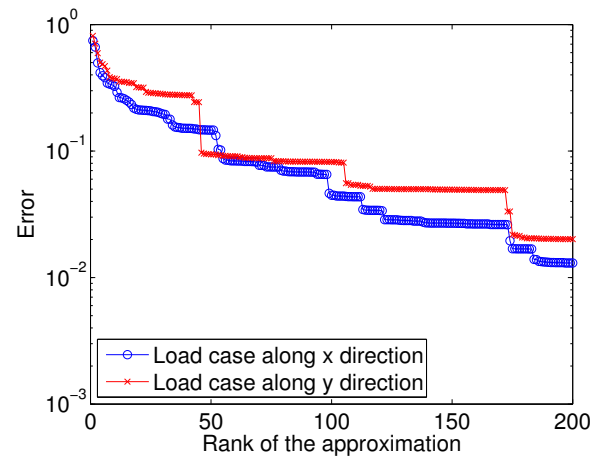

(c) EBC

Figure 5: Convergence of the progressive rank- $m$ PGD approximation with respect to rank $m$. Relative error in $L^{2}$ norm.

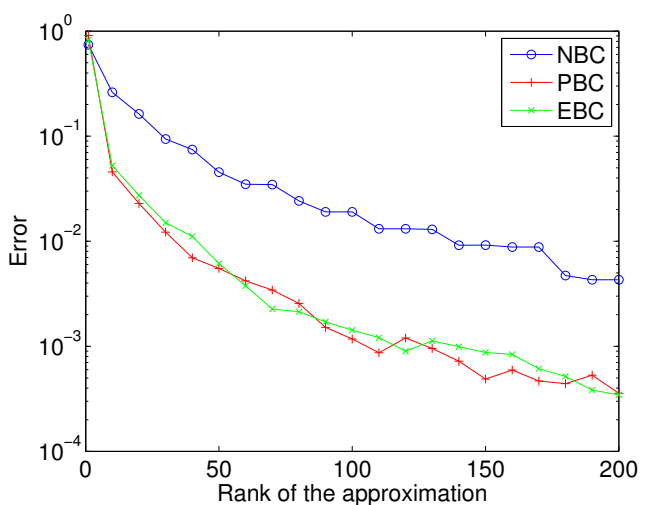

Figure 6: Relative error in canonical norm on the homogenized tensor as a function of the rank of the approximation 


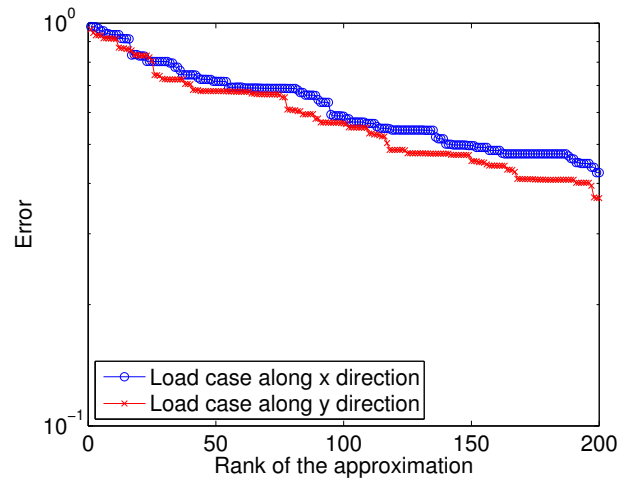

(a) $\mathrm{NBC}$

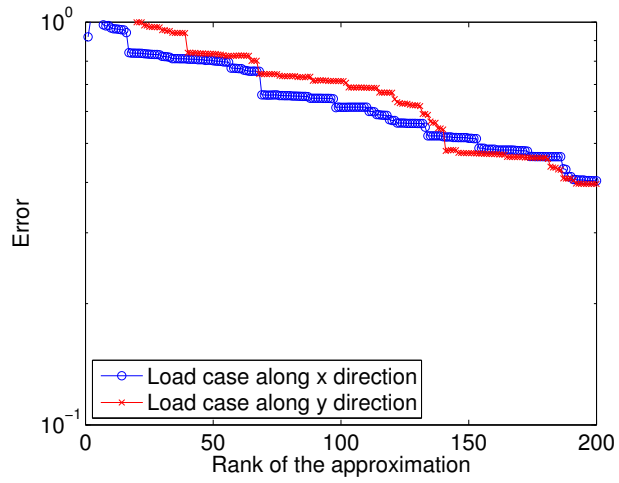

(b) $\mathrm{PBC}$

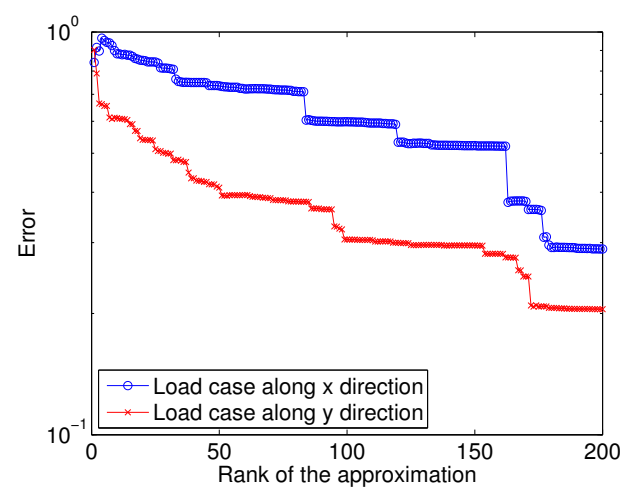

(c) $\mathrm{EBC}$

Figure 7: Convergence of the progressive rank- $m$ PGD approximation with respect to rank $m$. Relative error in $L^{2}$ norm, $k_{i}=1000 W \cdot m^{-1} . K^{-1}$.

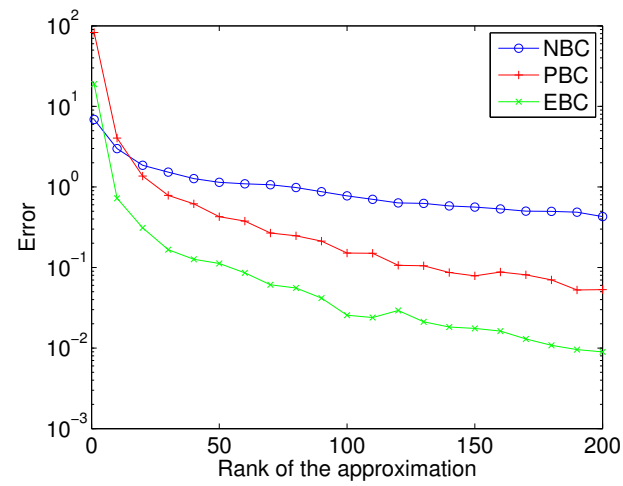

Figure 8: Relative error in canonical norm on the homogenized tensor as a function of the rank of the approximation. $k_{i}=1000 W \cdot m^{-1} \cdot K^{-1}$. 


\subsection{PGD using Tucker format}

In this section we compare the convergence between the progressive PGD with canonical tensor format (algorithm 1) with the progressive PGD with Tucker format (algorithm $3)$.

We consider the 3D image with $128^{3}$ voxels represented in figure 9 . This image is associated with a domain $\Omega=(0,1)^{3}$ which contains fibers in a matrix. Phases are isotropic with thermal conductivities $k_{f}=10 \mathrm{~W} \cdot \mathrm{m}^{-1} \cdot \mathrm{K}^{-1}$ for the fibers and $k_{m}=1 \mathrm{~W} \cdot \mathrm{m}^{-1} \cdot \mathrm{K}^{-1}$ for the matrix. The image has been separated using the Tucker ALS method from the

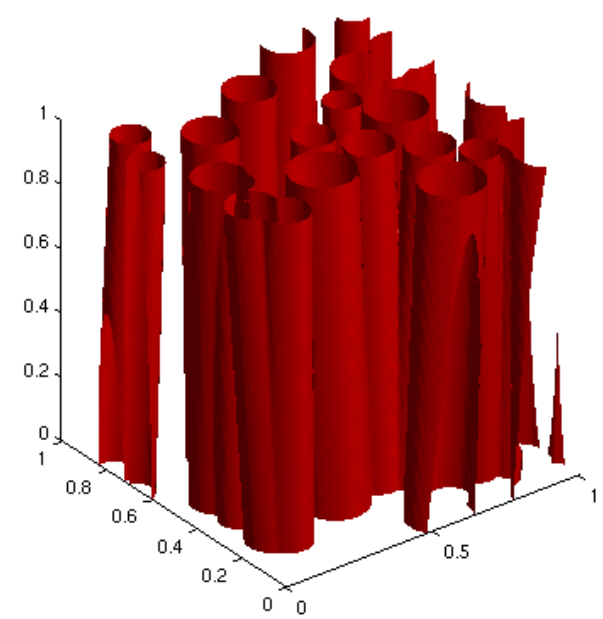

Figure 9: Random fibers into a matrix

MATLAB $^{\text {TM }}$ Tensor Toolbox [4] with a rank- $(119,119,18)$ Tucker decomposition, automatically taking into account the anisotropy of the original microstructure. The relative error in $L^{2}$ norm between the separated representation and the real characteristic function is lower than $1 \%$.

The two alternatives proposed in algorithms 1 and 3 have been tested with PBC, for the load case $\nabla u^{M}=(1,0,0)^{T}$. Here, the residual serves as an error indicator. It is plotted figure 10. This figure shows that the algorithm 3 converges faster than 1 . Indeed, while 40 iterations are needed to reach a residual of 0.0532 for algorithm 3 , algorithm 1 needs 90 iterations to reach the same value. However, algorithm 3 has a much higher computational cost than algorithm 1 due to the projection on the subspaces $\left(U_{m}\right)_{m \in \mathbb{N}^{*}}$. This limitation restricts the use of algorithm 3 to low dimensional cases (2 or 3 ).

Even if the residual has a poor convergence rate in both cases, we can see on figure 11 that the homogenized value $\underline{\underline{K}}_{x x}^{h}$ converges rapidly. The fast convergence of homogenized value in contrast with residual justifies the definition of new error indicators. They will be defined in the next section via goal oriented error estimation and the construction of adaptive algorithms for the solution of problem (19). 


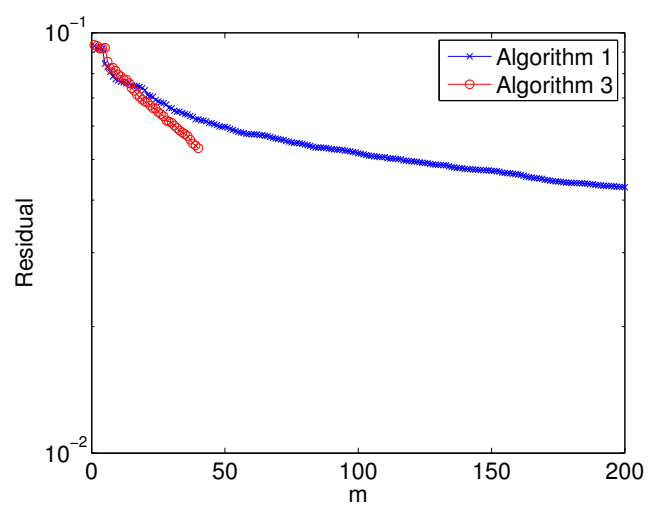

Figure 10: Residual norm with respect to $\mathrm{m}$. Comparison between canonical decomposition (algorithm 1) or Tucker representation (algorithm 3).

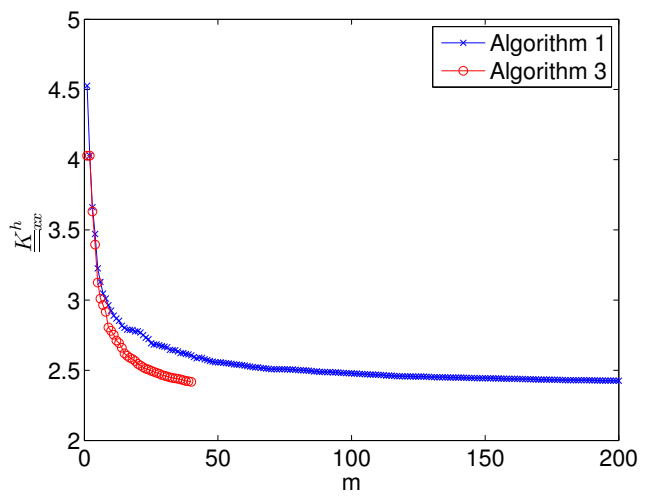

Figure 11: $\underline{\underline{K}}_{x x}^{h}$ with respect to $\mathrm{m}$. Comparison between canonical decomposition (algorithm 1) or Tucker representation (algorithm 3).

\section{Goal-oriented error estimation}

We consider the variational problem:

$$
\begin{aligned}
& \text { Find } u \in \mathcal{V} \text { such that } \\
& \forall \delta u \in \mathcal{V}, a(\delta u, u)=l(\delta u)
\end{aligned}
$$

We denote by $u_{m} \in \mathcal{V}$ an approximation of $u$ obtained with a PGD algorithm. The aim is here to provide a methodology for an adaptive approximation of the solution relying on the classical dual-primal error estimator $[1,2]$. The main difference with respect to [2] concerns the resolution of the dual problem: in this former contribution, the number of modes needed for solving this problem were pre-defined in advance. However, this number of modes is highly problem-dependent. In this contribution, an adaptive strategy is proposed in order to circumvent this issue and specific improvements related to the present class of applications are introduced.

The content of this section is the following: After presenting this adaptive method in 
a general context of linear quantities of interest, we provide a specific methodology for the present context of computational homogenization, where different linear quantities of interest (the coefficients of the homogenized tensor) have to be computed.

\subsection{Quantity of interest and adjoint problem}

We define a quantity of interest $Q(u)$ with $Q: \mathcal{V} \rightarrow \mathbb{R}$ a linear functional on $\mathcal{V}$. We introduce the adjoint problem

Find $\phi \in \mathcal{V}$ such that

$$
\forall \delta u \in \mathcal{V}, a(\delta u, \phi)=Q(\delta u)
$$

The quantity of interest can then be expressed $Q(u)=a(u, \phi)$. The error in the quantity of interest can be expressed:

$$
Q(u)-Q\left(u_{m}\right)=a\left(u-u_{m}, \phi\right)=l(\phi)-a\left(u_{m}, \phi\right)=\left\langle\phi, R\left(u_{m}\right)\right\rangle
$$

where $R\left(u_{m}\right)$ is the residual associated with the approximation $u_{m}$.

\subsection{Approximation of the adjoint problem}

Suppose that we have an approximation $\phi_{n}$ of $\phi$. We then have

$$
Q(u)-Q\left(u_{m}\right)=a\left(u-u_{m}, \phi-\phi_{n}\right)+a\left(u-u_{m}, \phi_{n}\right)
$$

If the primal and adjoint problems are solved with a sufficient accuracy, the first term in the right hand side can be neglected and we have the following estimation of the error in the quantity of interest:

$$
Q(u)-Q\left(u_{m}\right) \approx a\left(u-u_{m}, \phi_{n}\right)
$$

In practice, in order to guaranty a good estimation of the error, we can construct a sequence of approximations $\phi_{n}$ of $\phi$ until the estimation $a\left(u-u_{m}, \phi_{n}\right)$ has converged with a desired tolerance.

\subsection{Improvement of the estimation}

Note that the estimation $Q\left(u_{m}\right)$ converges with a convergence rate at least of the same order as $u_{m}$. Indeed,

$$
\left|Q(u)-Q\left(u_{m}\right)\right|=\left|a\left(u-u_{m}, \phi\right)\right| \leq C\left\|u-u_{m}\right\|\|\phi\|
$$

where $C$ is the continuity constant of $a$. After solving the adjoint problem and with no additional cost, we can introduce an improved estimation of the quantity of interest:

$$
\widehat{Q}\left(u_{m}, \phi_{n}\right)=Q\left(u_{m}\right)+a\left(u-u_{m}, \phi_{n}\right)
$$

We have

$$
\left|Q(u)-\widehat{Q}\left(u_{m}, \phi_{n}\right)\right|=\left|a\left(u-u_{m}, \phi-\phi_{n}\right)\right| \leq C\left\|u-u_{m}\right\|\left\|\phi-\phi_{n}\right\|
$$

Note that if the adjoint problem were solved exactly, $\widehat{Q}\left(u_{m}, \phi\right)$ would be equal to the exact quantity of interest $Q(u)$, even if $u_{m}$ is only an approximation of $u$. So in practice, the error analysis is also a way of improving the estimation of quantities of interest. 


\subsection{Adaptive approximation using error estimation}

Suppose that we have a rank- $m_{i}$ approximation $u_{m_{i}}$ of $u$. In order to estimate the error on the quantity of interest, one should look at the convergence of the sequence $\left(E_{n}^{m_{i}}\right)_{n \in \mathbb{N}^{*}}$ defined by $E_{n}^{m_{i}}=a\left(u-u_{m_{i}}, \phi_{n}\right)=\widehat{Q}\left(u_{m_{i}}, \phi_{n}\right)-Q\left(u_{m_{i}}\right)$, where $\phi_{n}$ is a sequence of approximations of $\phi$.

In practice, one has to find an estimation $\widetilde{E}_{i}$ of the true error $E_{i}$ defined by

$$
E_{i}=a\left(u-u_{m_{i}}, \phi\right)=\lim _{n \rightarrow \infty} E_{n}^{m_{i}}
$$

This estimator $\widetilde{E}_{i}$ is defined by $\widetilde{E}_{i}=E_{N_{i}}^{m_{i}}$, with $N_{i}$ such that

$$
\max _{j \in\left\{0, \ldots, j_{\max }\right\}} \frac{\left|E_{N_{i}+j+1}^{m_{i}}-E_{N_{i}+j}^{m_{i}}\right|}{\left|E_{N_{i}+j}^{m_{i}}\right|}<\varepsilon_{\text {stag }}
$$

with $\varepsilon_{\text {stag }}=10^{-2}$ a stagnation criterion. Note that this condition is chosen in order take into account possible oscillations of the sequence $\left\{E_{n}^{m_{i}}\right\}_{n}$. In practice, we choose $j_{\max }=9$.

If $\left|\widetilde{E}_{i}\right|$ is lower than a prescribed tolerance $\varepsilon_{t o l}, Q\left(u_{m_{i}}\right)$ is considered as a good estimation of $Q(u)$. Otherwise, we compute a rank- $m_{i+1}$ approximation $u_{m_{i+1}}$ of $u$ with $m_{i+1}=m_{i}+m_{\text {step }}$, we compute $\widetilde{E}_{i+1}$ and we compare it again to the prescribed tolerance. In this case, $u_{m_{i+1}}$ is computed starting from $u_{m_{i}}$ in a greedy fashion. The adaptive algorithm is summed up in algorithm 4 .

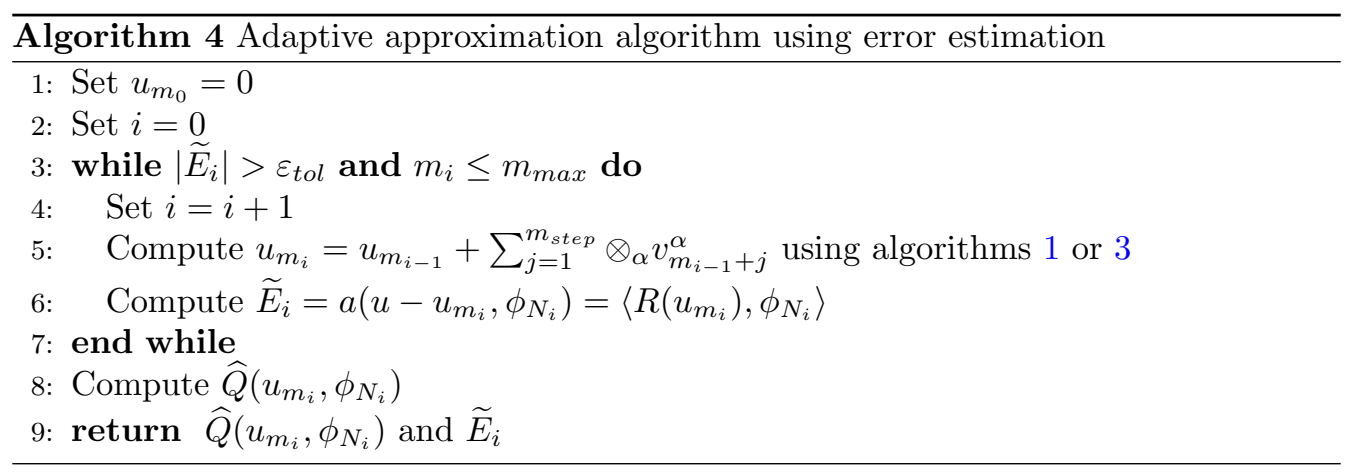

\subsection{Expression of the quantities of interest}

In the context of homogenization, the quantities of interest are the components of the homogenized tensor. They can be extracted using the following linear functionals:

$$
\left\{\begin{array}{l}
\text { NBC: } Q_{\alpha}(u)=\frac{1}{|\Omega|} \int_{\Omega} \underline{\nabla} u \cdot \underline{e_{\alpha}} d \Omega \\
\text { PBC, EBC: } Q_{\alpha}(u)=\frac{1}{|\Omega|} \int_{\Omega} \underline{\nabla} u \cdot \underline{\underline{K}} \cdot \underline{e_{\alpha}} d \Omega
\end{array}\right.
$$

where $\left\{\underline{e_{\alpha}}\right\}_{\alpha}$ is the canonical basis of $\mathbb{R}^{d}$, if the following values of $\underline{q}^{M}$ or $\underline{\nabla} u^{M}$ are used

$$
\left\{\begin{array}{l}
\text { NBC: } \underline{q}_{\alpha}^{M}=\underline{e}_{\alpha} \\
\text { PBC, EBC: }\left(\underline{\nabla} u^{M}\right)_{\alpha}=\underline{e}_{\alpha}
\end{array}\right.
$$


Indeed, for a given type of boundary conditions (NBC, $\mathrm{PBC}$ or $\mathrm{EBC}$ ), if we denote by $\left\{u_{\alpha}\right\}_{\alpha}$ the solutions of the $d$ boundary value problems associated with each load case $\alpha$, we have

$$
\left\{\begin{array}{l}
\text { NBC: }\left(\underline{\underline{K}}^{h}\right)_{\beta \alpha}^{-1}=Q_{\alpha}\left(u_{\beta}\right) \\
\text { PBC, EBC: } \underline{\underline{K}}_{\beta \alpha}^{h}=Q_{\alpha}\left(u_{\beta}\right)
\end{array}\right.
$$

We introduce the corresponding weak formulations

$$
a\left(\delta u, u_{\alpha}\right)=l_{\alpha}(\delta u) \quad \forall \delta u \in \mathcal{V}
$$

At the end, we have 6 problems to solve for a 3D problem, the 3 primal problems and the 3 adjoint problems.

\subsection{Adaptive approximation in $2 D$}

We consider homogenization with $\mathrm{EBC}$ on the microstructure represented in figure 2. The phases have homogeneous conductivities with conductivities $k_{m}=1 W \cdot m^{-1} \cdot K^{-1}$ for the matrix and $k_{i}=10 \mathrm{~W} \cdot \mathrm{m}^{-1} \cdot K^{-1}$ for the inclusions. The image $I$ contains $512 \times 512$ pixels and is associated with a domain $\Omega=(0,1)^{2}$. The image $I$ has been smoothed with a characteristic length of $\delta=2$ pixels. A SVD has been applied to obtain the separated representation $I_{s}$ of $I$ such that the relative error in $L^{2}$ norm is lower than $1 \%$. As a consequence, $I_{s}$ is a rank-309 approximation of $I$.

\subsubsection{Estimation of $K_{x x}^{h}$}

First, we are interested in $\underline{\underline{K}}_{x x}^{h}=Q_{x}\left(u_{x}\right)$. We apply the algorithm 4 to find a good approximation of this homogenized value. The parameters are $m_{\text {step }}=20$ and $m_{\max }=$ 400. The tolerance is fixed to $\varepsilon_{t o l}=10^{-2}$.

In the end, the estimated error is $\widetilde{E}_{3}=E_{45}^{60}=0.8710^{-2}$ and the estimated quantity of interest are $Q_{x}\left(u_{x, 60}\right)=1.79 \mathrm{~W} \cdot \mathrm{m}^{-1} \cdot K^{-1}$ and $\widehat{Q}_{x}\left(u_{x, 60}, u_{\phi, 45}\right)=1.80 \mathrm{~W} \cdot \mathrm{m}^{-1} \cdot \mathrm{K}^{-1}$. The convergence of $\left(\left|E_{n}^{m_{i}}\right|\right)_{n \in \mathbb{N}^{*}}$ is shown in figure 12 .

Note that we are interested in the right part of the curves, when the error estimator converges. The behavior of the curve $m_{i}=20$ around $n=13$ is explained by a change of sign in the estimated error.

\subsubsection{Estimation of $K_{x y}^{h}$}

We now look at $\underline{\underline{K}}_{x y}^{h}=Q_{y}\left(u_{x}\right)$. We apply again the algorithm 4 with $m_{\text {step }}=20$ and $m_{\max }=400$. However, given that the homogenized material is expected to be isotropic, we take $\varepsilon_{t o l}=10^{-3}$. Finally we get $\widetilde{E}_{2}=6.5610^{-4}$ which results in $Q_{y}\left(u_{x, 40}\right)=$ $0.9910^{-2} W \cdot m^{-1} \cdot K^{-1}$ and $\widehat{Q}_{y}\left(u_{x, 40}, \phi_{y, 45}\right)=1.0610^{-2} W \cdot m^{-1} \cdot K^{-1}$. Different error estimations for different iterations of the adaptive algorithm are plotted in figure 13. Figure 13 shows that the stagnation criterium proposed in (54) is too restrictive. This is due to the isotropy of the material and the particularly small value of $\underline{\underline{K}}_{x y}^{h}$. Indeed, if we relax the stagnation criterium by setting $\varepsilon_{\text {stag }}=10^{-1}$, we can see that for $m_{i}=40$ the rank $N_{i}$ of the adjoint approximation goes from 226 to 40 with a good approximation of the absolute error $\left|E_{N_{i}}^{m_{i}}\right|$. The latter goes from $7.7010^{-4}$ to $3.9910^{-4}$ so we still have a good estimation of the absolute error. The loss of precision is counterbalanced by much faster computations of the different estimates. 


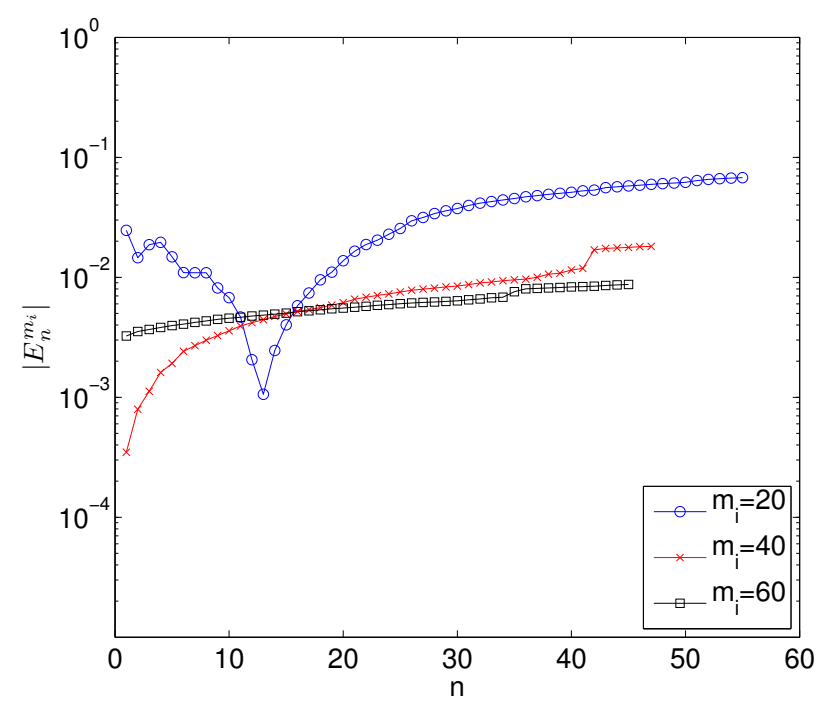

Figure 12: Error estimation as a function of the rank $n$ of the approximation of the adjoint solution $\phi_{x}$ when computing $\underline{\underline{K}}_{x x}^{h}=Q_{x}\left(u_{x}\right)$ with $\varepsilon_{\text {stag }}=10^{-2}$

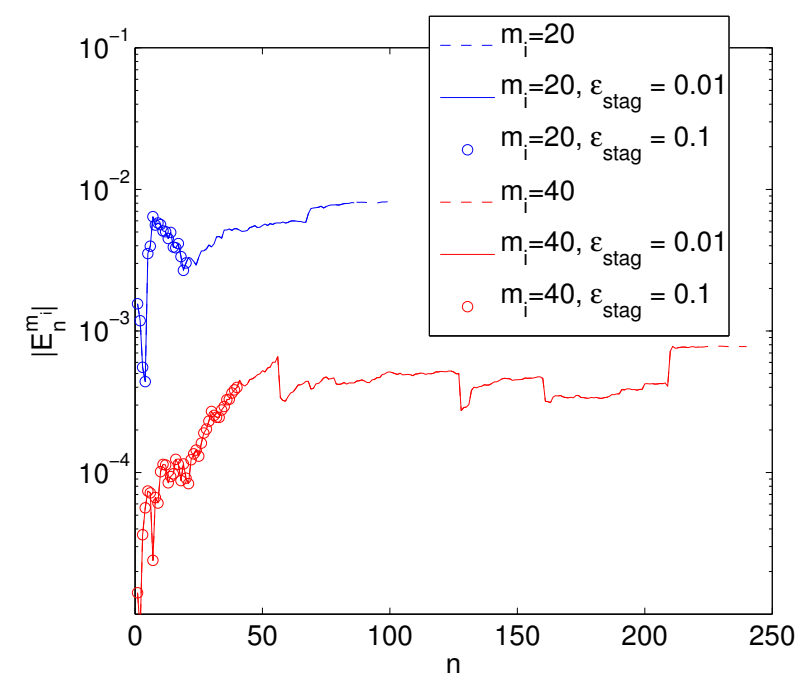

Figure 13: Error estimation as a function of the rank $n$ of the approximation of the adjoint solution $\phi_{y}$ when computing $\underline{\underline{K}}_{x y}^{h}=Q_{y}\left(u_{x}\right)$ with different $\varepsilon_{s t a g}$

\subsection{Specificities in the context of homogenization}

For NBC, noting that $\underline{q}_{\alpha}^{M}=\underline{e}_{\alpha}$ and using the Green formula we obtain

$$
Q_{\alpha}(u)=-\frac{1}{|\Omega|} l_{\alpha}(u)
$$


This equality also holds for PBC and EBC if $\underline{\nabla} u_{\alpha}^{M}=\underline{e}_{\alpha}$. Therefore, the following important property holds for every type of boundary conditions:

$$
\phi_{\alpha}=-\frac{1}{|\Omega|} u_{\alpha}
$$

for all $\alpha$. That means that for $d$-dimensional problems, we only need to solve $d$ problems instead of $2 \times d$ problems.

With these properties, a special adaptive algorithm can be derived for homogenization. First, the diagonal terms of the homogenized tensor $\underline{\underline{K}}_{\alpha \alpha}^{h}$ are determined using the relation (57). On one hand, supposing that we already have an approximation $u_{m}=\sum_{i=1}^{m} v_{i}$ of $u$, this relation implies that $\mathcal{V}_{m}=\operatorname{span}\left\{v_{i}\right\}_{i=1}^{m}$ is a good space for the approximation of $\phi$ through Galerkin projection. On the other hand, with $\phi_{m}=\sum_{i=1}^{m} v_{i}$ an approximation of $\phi, \mathcal{V}_{m}=\operatorname{span}\left\{v_{i}\right\}_{i=1}^{m}$ is also a good approximation space for the Galerkin projection of $\phi$. The algorithm for the estimation of the diagonal components of the homogenized tensor is written in algorithm 5 .

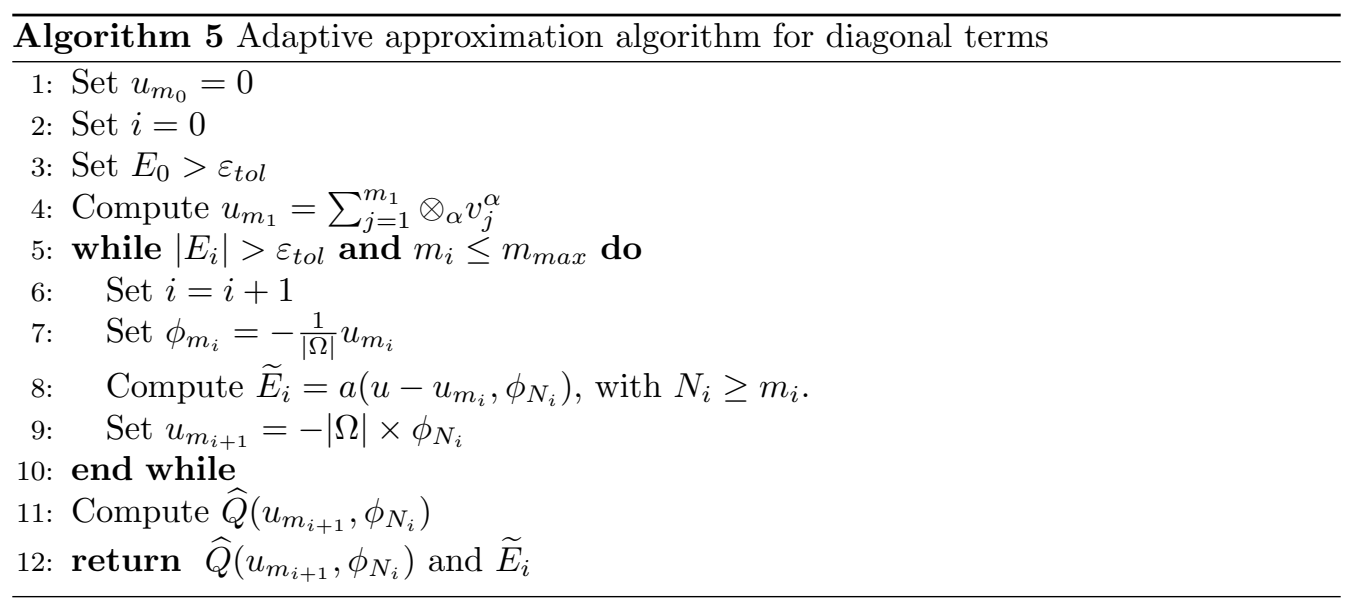

One can choose to solve only primal or adjoint problems for these steps. Then, given that we have already approximations $\left(u_{\alpha, m}\right), \forall \alpha$ and consequently $\left(\phi_{\alpha, m}\right), \forall \alpha$, we can have a first error estimation of non diagonal terms of the homogenized tensor $\underline{\underline{K}}_{\alpha \beta}^{h}, \forall(\alpha, \beta), \alpha \neq \beta$. If the error is too high, we can apply algorithm 4 to find an estimation of $\underline{\underline{K}}_{\alpha \beta}^{h}$. We denote $\widetilde{E}_{\alpha \beta}$ the error estimation on $\underline{\underline{K}}_{\alpha \beta}^{h}$. The adaptive algorithm is summed up in algorithm 6 .

\subsection{Remark on the approximation of the adjoint problem}

Note that the adjoint problem must be solved with a precision higher than the primal problem, in the following sense. Suppose that $u_{m}$ is the Galerkin projection of $u$ in a linear subspace $\mathcal{V}_{m} \subset \mathcal{V}$, that means

$$
\forall \delta u \in \mathcal{V}_{m}, \quad a\left(\delta u, u_{m}\right)=l(\delta u)
$$

If $\phi_{n}$ is defined as the Galerkin approximation of $\phi$ in $\mathcal{V}_{m}$, i.e. $\phi_{n}=\phi_{m}^{*}$ with

$$
\forall \delta u \in \mathcal{V}_{m}, \quad a\left(\delta u, \phi_{m}^{*}\right)=Q(\delta u)
$$




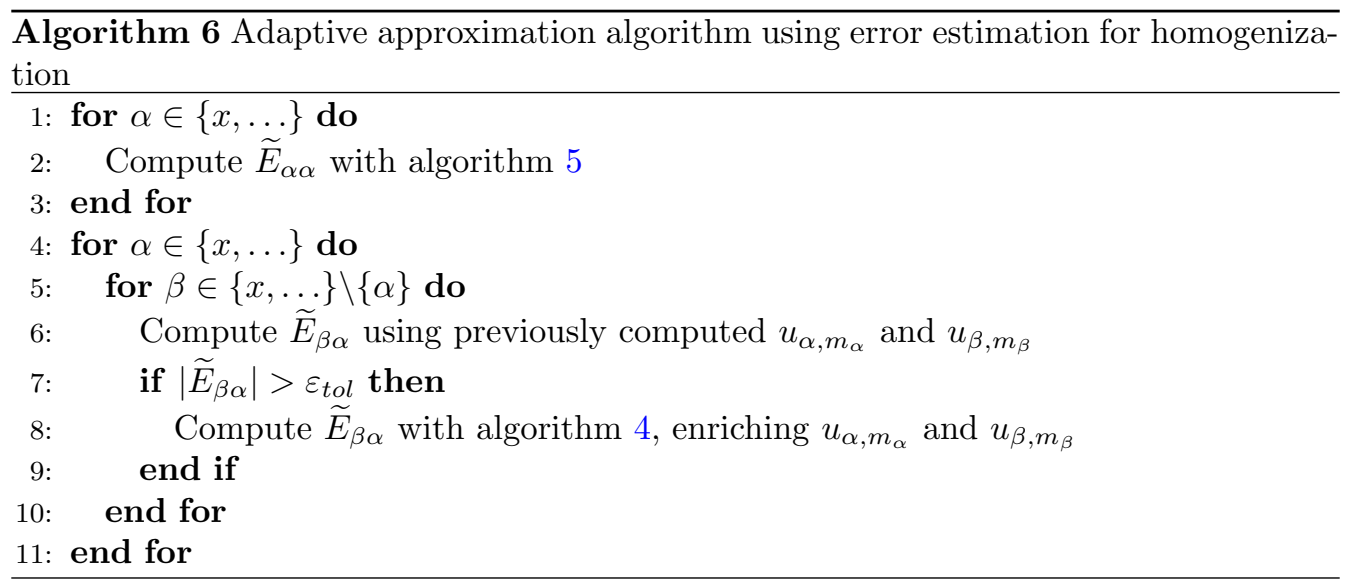

then,

$$
a\left(u-u_{m}, \phi_{n}\right)=0
$$

yielding $Q(u)-Q\left(u_{m}\right) \approx 0$, which is of course a bad estimation of the error. For example, when constructing a rank- $m$ approximation $u_{m}=\sum_{i=1}^{m} \lambda_{i} v_{i}$ with an update of coefficients $\lambda_{i}$, we have that $u_{m}$ is the Galerkin approximation of $u$ in $\mathcal{V}_{m}=\operatorname{span}\left\{v_{i}\right\}_{i=1}^{m}$. We then obtain a bad estimation of the error if we compute an approximation $\phi_{n}$ in $\mathcal{V}_{m}$.

A possible remedy consists in defining $\phi_{n}=\phi_{m}^{*}+\delta \phi_{m, n}$ with $\delta \phi_{m, n}$ an approximation of $\delta \phi_{m}=\phi-\phi_{m}^{*}$ which is the solution of

$$
a\left(\delta u, \delta \phi_{m}\right)=Q(\delta u)-a\left(\delta u, \phi_{m}^{*}\right) \quad \forall \delta u \in \mathcal{V}
$$

Note that if $\phi_{m}^{*}=\sum_{i=1}^{m} \mu_{i} v_{i}$ and $\delta \phi_{m, n}=\sum_{i=m+1}^{n} \mu_{i} v_{i}$, we can improve the approximation $\phi_{n}$ by defining it as the Galerkin projection of $\phi$ in $\mathcal{V}_{n}=\operatorname{span}\left\{v_{i}\right\}_{i=1}^{n} \supset \mathcal{V}_{m}$.

As mentioned above, whatever the approximation strategy for the adjoint problem, if we are able to construct a sequence of approximations $\phi_{n}$, the convergence of the quantity $a\left(u-u_{m}, \phi_{n}\right)$ should be checked and compared to $Q\left(u_{m}\right)$.

\section{Thermal homogenization of ductile cast iron}

The last application concerns a segmented image that represents a sample of a ductile cast iron. The image has been obtained by Computed Tomography (CT) and it contains $128^{3}$ voxels of size $2.2833 \mu \mathrm{m}$. The material is composed of iron and graphite. The indicator function of the graphite's phase is plotted in figure 14. The conductivities are $k_{i}=76.2 \mathrm{~W} \cdot \mathrm{m}^{-1} \cdot \mathrm{K}^{-1}$ for the iron phase and $k_{g}=24.0 \mathrm{~W} \cdot \mathrm{m}^{-1} \cdot \mathrm{K}^{-1}$ for the carbon phase. It turns out that the characteristic function $I$ of the graphite phase is hardly separable. As a consequence, we describe the exact geometry with the specific Tucker representation introduced in (45) with a minimal linear smoothing on one element. The algorithm 6 is employed. The homogenization with the PBC is applied. The tolerance on the components of the conductivity tensor is set to $0.1 \mathrm{~W} \cdot \mathrm{m}^{-1} \cdot \mathrm{K}^{-1}$. We choose a 


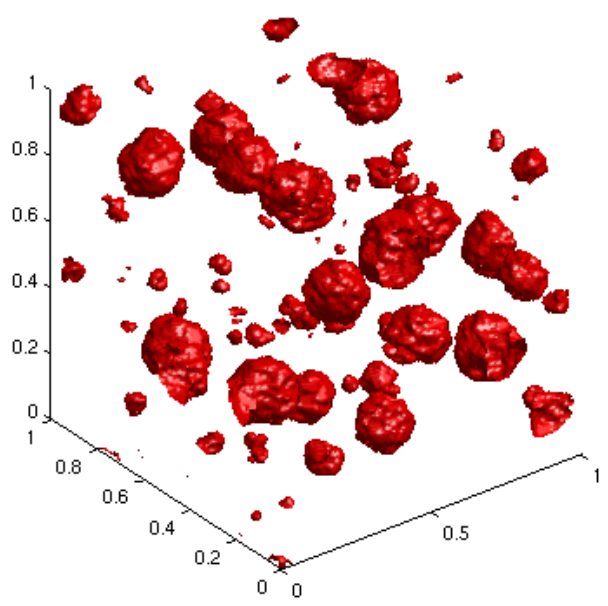

Figure 14: Ductile cast iron, characteristic function of the graphite phase

stagnation criterium $\varepsilon_{\text {stag }}^{\alpha \beta}$ for the computation of the error indicator defined by

$$
\varepsilon_{\text {stag }}^{\alpha \beta}=\left\{\begin{array}{l}
10^{-2} \text { if } \alpha=\beta \\
10^{-1} \text { otherwise }
\end{array}\right.
$$

as we expect that the material is isotropic. $u_{\alpha}$ is increased by rank-50 tensor at each step of the adaptive algorithm.

From a numerical point of view, only rank-100 approximations of the solutions of the different loadings were necessary to get the diagonal components $\underline{\underline{K}}_{\alpha \alpha}^{h}, \alpha \in\{x, \ldots, z\}$ with the tolerance of $0.1 W \cdot m^{-1} \cdot K^{-1}$. However, rank-350 (resp. rank-300, rank-250) approximations of the adjoint solutions was needed to obtain the error estimate of $\underline{\underline{K}}_{x y}^{h}$ (resp. $\underline{\underline{K}}_{x z}^{h}, \underline{\underline{K}}_{y z}^{h}$ ) with the desired accuracy.

In the end, the estimated homogenized conductivity is

$$
\underline{\underline{K}}^{h}=\left(\begin{array}{cccc}
73.35 & 1.810^{-3} & -4.5 & 10^{-3} \\
& 73.36 & -5.6 & 10^{-4} \\
\text { sym } & & 73.36
\end{array}\right)
$$

with estimated componentwise absolute error

$$
|\widetilde{E}|=\left(\begin{array}{ccccc}
7.510^{-2} & 4.7 & 10^{-4} & 3.9 & 10^{-4} \\
& 8.7 & 10^{-2} & 5.2 & 10^{-4} \\
\text { sym } & & & 9.3 & 10^{-2}
\end{array}\right)
$$

As expected, the material appears to be isotropic. Note that the obtained precision is better than the one prescribed in the adaptive algorithm. This is due to the fact that the error associated with an approximation $u_{m}$ is not estimated at each iteration $m$ but only every 50 iterations. A reference finite element computation provides the following 
homogenized tensor

$$
\underline{\underline{K}}_{F E M}^{h}=\left(\begin{array}{ccc}
73.16 & 3.610^{-4} & -4.510^{-3} \\
& 73.19 & 5.710^{-4} \\
\text { sym } & & 73.20
\end{array}\right)
$$

The true componentwise absolute error is

$$
\left|\widetilde{E}_{\text {true }}\right|=\left(\begin{array}{ccccc}
1.910^{-1} & 1.4 & 10^{-3} & 7.7 & 10^{-5} \\
& 1.7 & 10^{-1} & 1.1 & 10^{-3} \\
\text { sym } & & & 1.6 & 10^{-1}
\end{array}\right)
$$

As we can see, the estimated error underestimates the true error (except for the $x z$ component) but provides a very good estimation of this true error.

Remark 8. Negative values for the homogenized tensors are meaningless. They are in fact almost null values so we can set them to 0 .

Remark 9. For very large images, the methodology can be directly applied but practical issues have to be addressed in order to overcome memory limitations due to the representation of the geometry. In practice, the image should be decomposed into sub-images and tensor approximation methods applied on these different sub-images. This way, the operator in Tucker tensor format is decomposed in smaller operators associated to each sub-image.

\section{Conclusion}

A complete numerical strategy based on tensor approximations has been proposed for image-based numerical homogenization. We have first introduced some specific treatments of the geometry, including a smoothing of indicator functions in order to obtain accurate low-rank representations of the geometrical data. This yields a formulation of boundary value problems in a suitable tensor format. Suitable weak formulations of boundary value problems that preserve tensor format have been introduced in order to handle the different types of boundary conditions that are used in classical numerical homogenization methods. Then, some variants of Proper Generalized Decomposition (PGD) methods have been introduced for the a priori construction of tensor decompositions of the solution of boundary value problems. In particular, a new definition of PGD has been proposed for the progressive construction of a decomposition of the solution in Tucker format. This tensor format appears to be well adapted to the present application and the proposed algorithms yields a rapid convergence of tensor decompositions. Finally, a dual-based error estimation strategy has been considered in order to assess the quality of the prediction of homogenized properties. A goal oriented adaptive strategy has been proposed for the construction of tensor approximations that satisfy a prescribed accuracy on predicted homogenized properties. The number of modes needed for the dual problem is obtained automatically by the adaptive strategy. The complete methodology has been successfully applied to images of real 2 or 3-dimensional microstructures.

Considering the computational time, figure 15 is of interest. A 3D problem has been solved for several resolutions using FEM and PGD approaches. The FEM solution is 
used as a reference for the computation of the $L^{2}$ relative error $\varepsilon$. The time reference is the computational time of the FEM solution on the image with the largest number of voxels. The operator used for the PGD is treated as in section 7, meaning that the whole picture has been taken into account, without truncation. We can see on figure 15 that for

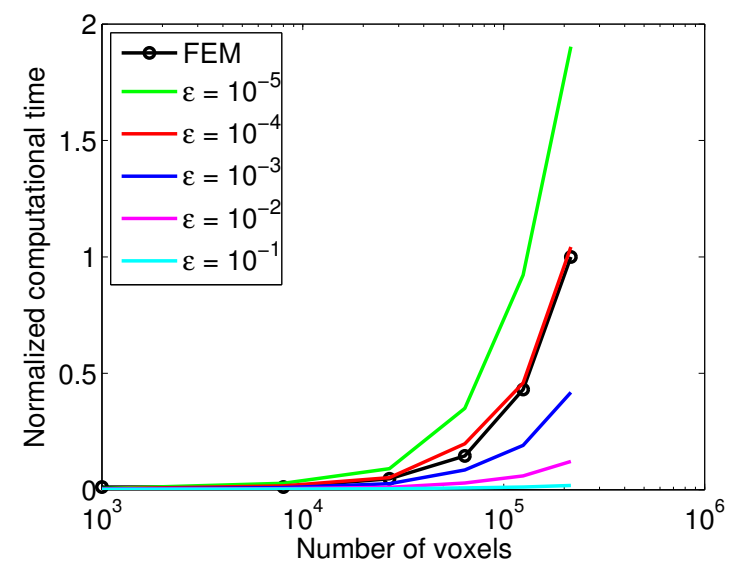

Figure 15: Normalized computational time as a function of the number of voxels and the relative error $\varepsilon$ in $L^{2}$ norm

a precision better than $10^{-4} \mathrm{FEM}$ is more interesting than PGD approaches. However looking for such a precision is meaningless since errors done in the model should be larger than $10^{-2}$ because of the image processing technique. An other advantage of the separate representation in term of computation is the low memory requirement. Indeed since only small 1D domains are considered, we do not have to manage very large stiffness matrices.

In order to go further in this work, additional parameters will be added to the solution such as the conductivity of each phase of the heterogeneous material. However to solve these high dimensional PDEs other tensor formats can be employed, in particular hierarchical Tucker tensor format and its derivations [19, 47]. Indeed the canonical decomposition has several drawbacks as discussed by de Silva and Lim in [52] especially in terms of existence, quality of the approximation and the so called "cancellation" effect. The orthogonality of the vectors in the Tucker tensors avoids these problems but this decomposition is still suffering from the curse of dimensionality since the size of the core tensor is growing with $r^{d}$ with $r=r^{x}=r^{y}=\ldots$. The hierarchical Tucker tensor format writes the core tensor as a hierarchy of smaller orders such that the linearity dependance with the dimension is recovered while keeping the orthogonality features of the Tucker decomposition.

\section{References}

[1] Ainsworth, M., Oden, J.T., 2000. A Posteriori Error Estimation in Finite Element Analysis. WileyInterscience. 1st edition.

[2] Ammar, A., Chinesta, F., Diez, P., Huerta, A., 2010. An error estimator for separated representations of highly multidimensional models. Computer Methods in Applied Mechanics and Engineering 199, 1872-1880. 
[3] Babin, P., Valle, G., Dendievel, R., Lassoued, N., Salvo, L., 2005. Mechanical properties of bread crumbs from tomography based Finite Element simulations. Journal of Materials Science 40, 58675873.

[4] Bader, B., Kolda, T., 2011. Matlab tensor toolbox version 2.4. http://csmr.ca.sandia.gov/ tgkolda/TensorToolbox/.

[5] Bensoussan, A., Lions, J., Papanicolaou, G., 1978. Asymptotic Analysis for Periodic Structures. North-Holland.

[6] Camacho, D.L.A., Hopper, R.H., Lin, G.M., Myers, B.S., 1997. An improved method for finite element mesh generation of geometrically complex structures with application to the skullbase. Journal of Biomechanics 30, 1067 - 1070.

[7] Cances, E., Ehrlacher, V., Lelievre, T., 2011. Convergence of a greedy algorithm for highdimensional convex nonlinear problems. Mathematical Models \& Methods In Applied Sciences 21, 2433-2467.

[8] Carroll, J.D., Chang, J., 1970. Analysis of individual differences in multidimensional scaling via an n-way generalization of "Eckart-Young" decomposition. Psychometrika 35, 283-319.

[9] Charras, G.T., Guldberg, R.E., 2000. Improving the local solution accuracy of large-scale digital image-based finite element analyses. Journal of Biomechanics 33, $255-259$.

[10] Chinesta, F., Ammar, A., Cueto, E., 2010. Recent advances in the use of the Proper Generalized Decomposition for solving multidimensional models. Archives of Computational Methods in Engineering 17, 327-350.

[11] Chinesta, F., Ammar, A., Lemarchand, F., Beauchene, P., Boust, F., 2008. Alleviating mesh constraints: Model reduction, parallel time integration and high resolution homogenization. Computer Methods in Applied Mechanics and Engineering 197, 400-413.

[12] Chinesta, F., Ladeveze, P., Cueto, E., 2011. A short review on model order reduction based on proper generalized decomposition. Archives of Computational Methods in Engineering 18, 395-404.

[13] Duster, A., Parvizian, J., Yang, Z., Rank, E., 2008. The finite cell method for three-dimensional problems of solid mechanics. Computer Methods in Applied Mechanics and Engineering 197, 37683782 .

[14] Falco, A., Hackbusch, W., submitted. On minimal subspaces in tensor representations. Foundations of Computational Mathematics.

[15] Falco, A., Nouy, A., 2011. A proper generalized decomposition for the solution of elliptic problems in abstract form by using a functional Eckart-Young approach. Journal of Mathematical Analysis and Applications 376, 469-480.

[16] Falcó, A., Nouy, A., 2012. Proper generalized decomposition for nonlinear convex problems in tensor Banach spaces. Numerische Mathematik doi:10.1007/s00211-011-0437-5.

[17] Golanski, D., Terada, K., Kikuchi, N., 1997. Macro and micro scale modeling of thermal residual stresses in metal matrix composite surface layers by the homogenization method. Computational Mechanics 19, 188-202.

[18] Hackbusch, W., 2012. Tensor Spaces and Numerical Tensor Calculus. Springer. 2012 edition.

[19] Hackbusch, W., Kühn, S., 2009. A new scheme for the tensor representation. Journal of Fourier Analysis and Applications 15, 706-722.

[20] Harshman, R.A., 1970. Foundations of the PARAFAC procedure: Model and conditions for an" explanatory"multi-mode factor analysis. UCLA Working Papers in Phonetics 16, 1-84.

[21] Hollister, S., Kikuchi, N., 1994. Homogenization theory and digital imaging: a basis for studying the mechanics and design principles of bone tissue. Biotechnology and Bioengineering 43, 586-596.

[22] Jiang, M., Jasiuk, I., Ostoja-Starzewski, M., 2002. Apparent thermal conductivity of periodic two-dimensional composites. Computational Materials Science 25, 329-338.

[23] Kanit, T., Forest, S., Galliet, I., Mounoury, V., Jeulin, D., 2003. Determination of the size of the representative volume element for random composites: statistical and numerical approach. International Journal of Solids and Structures 40, 3647-3679.

[24] Kanit, T., N'Guyen, F., Forest, S., Jeulin, D., Reed, M., Singleton, S., 2006. Apparent and effective physical properties of heterogeneous materials: Representativity of samples of two materials from food industry. Computer Methods in Applied Mechanics and Engineering 195, 3960-3982.

[25] Keyak, J., Meagher, J., Skinner, H., Mote, C., Jr, 1990. Automated three-dimensional finite element modelling of bone: a new method. Journal of Biomedical Engineering 12, 389 - 397.

[26] Khoromskij, B., 2009. Tensor-Structured preconditioners and approximate inverse of elliptic operators in r^d. Constructive Approximation 30, 599-620.

[27] Kolda, T.G., Bader, B.W., 2009. Tensor decompositions and applications. SIAM Review 51, 455.

[28] Ladevèze, P., Passieux, J., Néron, D., 2010. The LATIN multiscale computational method and 
the Proper Generalized Decomposition. Computer Methods in Applied Mechanics and Engineering 199, 1287-1296.

[29] Ladevèze, P., Chamoin, L., 2011. On the verification of model reduction methods based on the proper generalized decomposition. Computer Methods in Applied Mechanics and Engineering 200, $2032-2047$.

[30] Lamari, H., Ammar, A., Cartraud, P., Chinesta, F., Jacquemin, F., Legrain, G., 2010a. Recent advances in material homogenization. International Journal of Material Forming 3, 899-902.

[31] Lamari, H., Ammar, A., Cartraud, P., Legrain, G., Chinesta, F., Jacquemin, F., 2010b. Routes for efficient computational homogenization of nonlinear materials using the proper generalized decompositions. Archives of Computational Methods in Engineering 17, 373-391.

[32] Langville, A.N., Stewart, W.J., 2004. A kronecker product approximate preconditioner for SANs. Numerical Linear Algebra with Applications 11, 723-752.

[33] Laschet, G., 2002. Homogenization of the thermal properties of transpiration cooled multi-layer plates. Computer Methods in Applied Mechanics and Engineering 191, 4535-4554.

[34] Lathauwer, L., Moor, B., Vandewalle, J., 2000. A multilinear singular value decomposition. SIAM J. MATRIX ANAL. APPL. 21, 1253-1278.

[35] Legrain, G., Allais, R., Cartraud, P., 2011a. On the use of the extended finite element method with quatree/octree meshes. International Journal for Numerical Methods in Engineering 86, 717-743.

[36] Legrain, G., Cartraud, P., Perreard, I., Moës, N., 2011b. An x-fem and level set computational approach for image-based modelling: Application to homogenization. International Journal for Numerical Methods in Engineering 86, 915-934.

[37] Legrain, G., Chevaugeon, N., Dréau, K., 2012. High order x-fem and levelsets for complex microstructures: Uncoupling geometry and approximation. Computer Methods in Applied Mechanics and Engineering 241 - 244, $172-189$.

[38] Lewis, A., Geltmacher, A., 2006. Image-based modeling of the response of experimental 3d microstructures to mechanical loading. Scripta Materialia 55, $81-85$.

[39] Lian, W., 2011. Contribution à l'Homogénéisation Numérique du Comportement Elastique de Matériaux à Microstructure Complexe Caractérisés par Imagerie. Ph.D. thesis. Ecole Centrale Nantes. Nantes.

[40] Lian, W.D., Legrain, G., Cartraud, P., 2012. Image-based computational homogenization and localization: comparison between X-FEM/levelset and voxel-based approaches. Computational Mechanics, Submitted.

[41] Madi, K., Forest, S., Boussuge, M., Gailliégue, S., Lataste, E., Buffiére, J.Y., Bernard, D., Jeulin, D., 2007. Finite element simulations of the deformation of fused-cast refractories based on x-ray computed tomography. Computational Materials Science 39, $224-229$.

[42] Maire, E., Fazekas, A., Salvo, L., Dendievel, R., Youssef, S., Cloetens, P., Letang, J.M., 2003. X-ray tomography applied to the characterization of cellular materials. related finite element modeling problems. Composites Science and Technology 63, $2431-2443$.

[43] Mishnaevsky Jr., L.L., 2005. Automatic voxel-based generation of 3d microstructural fe models and its application to the damage analysis of composites. Materials Science and Engineering: A 407, 11 -23 .

[44] Moulinec, H., Suquet, P., 1998. A numerical method for computing the overall response of nonlinear composites with complex microstructure. Computer Methods in Applied Mechanics and Engineering $157,69-94$

[45] Nouy, A., 2010a. A priori model reduction through proper generalized decomposition for solving time-dependent partial differential equations. Computer Methods in Applied Mechanics and Engineering 199, 1603-1626.

[46] Nouy, A., 2010b. Proper Generalized Decompositions and separated representations for the numerical solution of high dimensional stochastic problems. Archives of Computational Methods in Engineering 17, 403-434.

[47] Oseledets, I.V., 2011. Tensor-Train decomposition. SIAM Journal on Scientific Computing 33, 2295.

[48] Ostoja-Starzewski, M., 2006. Material spatial randomness: From statistical to representative volume element. Probabilistic Engineering Mechanics 21, 112-132.

[49] Ozdemir, I., Brekelmans, W., Geers, M., 2008. Computational homogenization for heat conduction in heterogeneous solids. International Journal for Numerical Methods in Engineering 73, 185-204.

[50] Sanchez-Palencia, E., 1980. Non homogeneous media and vibration theory. volume 127 of Lecture Notes in Physics. Springer Verlag, Berlin.

[51] Sethian, J., 1999. Level Set Methods: Evolving Interfaces in Geometry, Fluid Mechanics, Computer Vision and Materials Sciences. Cambridge Monographs on Applied and Computational Mathematics 
(No. 3), Cambridge University Press. 2nd edition.

[52] de Silva, V., Lim, L., 2008. Tensor rank and the Ill-Posedness of the best Low-Rank approximation problem. SIAM Journal on Matrix Analysis and Applications 30, 1084.

[53] Takano, N., Zako, M., Kubo, F., Kimura, K., 2003. Microstructure-based stress analysis and evaluation for porous ceramics by homogenization method with digital image-based modeling. International Journal of Solids and Structures 40, $1225-1242$

[54] Terada, K., Asai, M., Yamagishi, M., 2003. Finite cover method for linear and non-linear analyses of heterogeneous solids. International journal for numerical methods in engineering 58, 1321-1346.

[55] Touzene, A., 2008. A tensor sum preconditioner for stochastic automata networks. INFORMS Journal on Computing 20, 234-242.

[56] Ulrich, D., van Rietbergen, B., Weinans, H., Rüegsegger, P., 1998. Finite element analysis of trabecular bone structure: a comparison of image-based meshing techniques. Journal of Biomechanics $31,1187-1192$.

[57] Young, P., Beresford-West, T., Coward, S., Notarberardino, B., Walker, B., Abdul-Aziz, A., 2008. An efficient approach to converting three-dimensional image data into highly accurate computational models. Philosophical Transactions of the Royal Society A: Mathematical, Physical and Engineering Sciences 366, 3155-3173. 